\title{
Direct numerical simulation of hypersonic boundary layer transition over a blunt cone with a small angle of attack
}

\author{
Xinliang $\mathrm{Li}^{1},{ }^{1, \mathrm{a})}$ Dexun $\mathrm{Fu}^{2}$ and Yanwen $\mathrm{Ma}^{2}$ \\ ${ }^{1}$ LHD, Institute of Mechanics, Chinese Academy of Sciences, Beijing 100190, China \\ ${ }^{2}$ LNM, Institute of Mechanics, Chinese Academy of Sciences, Beijing 100190, China
}

(Received 30 July 2009; accepted 13 January 2010; published online 25 February 2010)

\begin{abstract}
The direct numerical simulation of boundary layer transition over a $5^{\circ}$ half-cone-angle blunt cone is performed. The free-stream Mach number is 6 and the angle of attack is $1^{\circ}$. Random wall blow-and-suction perturbations are used to trigger the transition. Different from the authors' previous work [Li et al., AIAA J. 46, 2899 (2008)], the whole boundary layer flow over the cone is simulated (while in the author's previous work, only two $45^{\circ}$ regions around the leeward and the windward sections are simulated). The transition location on the cone surface is determined through the rapid increase in skin fraction coefficient $\left(C_{f}\right)$. The transition line on the cone surface shows a nonmonotonic curve and the transition is delayed in the range of $20^{\circ} \leq \theta \leq 30^{\circ}\left(\theta=0^{\circ}\right.$ is the leeward section). The mechanism of the delayed transition is studied by using joint frequency spectrum analysis and linear stability theory (LST). It is shown that the growth rates of unstable waves of the second mode are suppressed in the range of $20^{\circ} \leq \theta \leq 30^{\circ}$, which leads to the delayed transition location. Very low frequency waves (VLFWs) are found in the time series recorded just before the transition location, and the periodic times of VLFWs are about one order larger than those of ordinary Mack second mode waves. Band-pass filter is used to analyze the low frequency waves, and they are deemed as the effect of large scale nonlinear perturbations triggered by LST waves when they are strong enough. (c) 2010 American Institute of Physics. [doi:10.1063/1.3313933]
\end{abstract}

\section{INTRODUCTION}

Correctly predicting transition locations is of great importance for the aerodynamic designs, thermal protections, and flying control of hypersonic vehicles. In recent years, boundary layer transition control becomes a powerful method for drag reduction, lift enhancement, noise reduction, and decreasing thermal load for flying vehicles. Well understanding the transition mechanism of hypersonic boundary layer is the foundation of transition control. However, the transition mechanism of hypersonic boundary layer is still not very clear until now.

Compared with incompressible boundary layer, transition mechanisms of hypersonic boundary layers are much more complex. Incompressible flat-plate boundary layer is always inviscous stable because there is no inflection point in the Blasius velocity profile. However, the compressible boundary layer can be inviscous unstable because its profile has generalized inflection points (GIPs). ${ }^{1}$ According to Mack's theory, ${ }^{2}$ in addition to the first instability mode (counterpart of T-S waves), there are higher acoustic instability modes with higher frequencies in supersonic and hypersonic boundary layers. In contrast to the unstable oblique first mode in low speed boundary layer flow, the twodimensional second Mack mode dominates in hypersonic boundary layers, which has been confirmed by the experiments of hypersonic boundary-layer flows over sharp or blunt cones. ${ }^{3-5}$ Stetson et al. ${ }^{4}$ carried out experiments to study the hypersonic boundary layer stability of a blunt cone

${ }^{a)}$ Electronic mail: lixl@imech.ac.cn. with $7^{\circ}$ half-angle and zero angle of attack, and fluctuation spectra at different locations were measured. Stetson's experiments show that the unstable second modes are dominant disturbance waves with significant superharmonic waves. But it is not unconditionally true that Mack second modes dominate the transition of hypersonic flow. By using the $e^{N}$ method, Su et al. ${ }^{6}$ found that the Mack second modes dominate the transition of an axisymmetrical Mach 6 blunt cone boundary layer with isothermal wall boundary, but the first modes dominate the transition for the same blunt cone flow with adiabatic wall boundary. Compared with axisymmetrical flow, hypersonic boundary layer flows over cones with nonzero angle of attack are more complex, and stability characteristics are different in different meridian planes. The study of transition mechanism of hypersonic cones with angle of attack is still insufficient.

As typical flows of hypersonic vehicles, hypersonic boundary layer transition flows over blunt cones are of great interest to aerospace engineering. Lots of wind tunnel and flight experiments are performed to measure the transition locations on supersonic/hypersonic blunted or sharp cone boundary layers. ${ }^{7,8}$ These experimental results show that transition onset Reynolds numbers for flight experiments are much larger than that for the wind tunnel ones, and the tunnel noise is deemed as the main reason. Horvath et al. ${ }^{8}$ conducted experimental investigations on $5^{\circ}$ half-angle cones in a conventional wind tunnel and a Mach 6 quiet wind tunnel, and the influence of tunnel noise was studied by comparing the transition onset locations obtained from convectional tunnel test with that obtained from quiet tunnel test. The comparison shows that the transition onset Reynolds number un- 


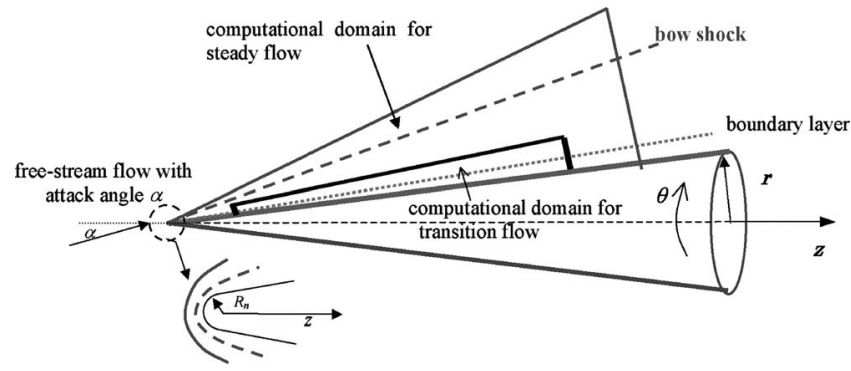

FIG. 1. Schematic of boundary layer flow over a blunt cone with angle of attack.

der low disturbance conditions was a factor of 1.3-1.6 greater than that measured in the conventional hypersonic tunnel or run in the quit tunnel run "noisy." In addition, leading bluntness, angle of attack, and wall roughness effects on transition onset location are also discussed in the report of Horvath $\mathrm{et} \mathrm{al}^{8}{ }^{8}$ Experimental results show that leading bluntness affects the transition location significantly. The increase in leading bluntness can retard the transition location, but the effect will be reversed when the bluntness exceeds a threshold. The effect of bluntness on the transition location is considered to associate with the entropy layer generated by the leading bow shock. The true mechanism, however, is still not clear now.

Until now, there is still no method capable of predicting transition locations accurately without relying on empirical parameters. The most widely used transition prediction method is the $e^{N}$ method that suggests that the transition occurs when the amplitude of most unstable disturbance grows $e^{N}$ times. Here $N$, usually between 9 and 11 for incompressible boundary layer, is an empirical number from experiments. As addressed in Zhong et al. ${ }^{9}$ the limit of $e^{N}$ method is that it does not consider the initial amplitude of disturbance, while the transition process depends on the initial disturbance. The properties of initial disturbance, including the amplitude, frequencies, wave number, and other parameters, can be better understood through the study of receptivity. Su et al. ${ }^{10}$ developed a modified $e^{N}$ method. In their method, both amplify and decay processes of the disturbance waves are considered, while only the growth process of unstable waves is considered in the conventional $e^{N}$ method. Su et al. ${ }^{10}$ calculated the transition locations of hypersonic boundary layer over a blunt cone with free-stream Mach number 6 . Their results agree with the current direct numerical simulation (DNS) data.

DNS becomes a more and more important tool in the study of turbulent flows and transitions. ${ }^{11}$ Because of complexity, the DNS of the compressible turbulent boundary layers started just recently. Compared with the DNS of flat-plate boundary layers, studies on the DNS of supersonic/ hypersonic boundary layers over cones are seldom reported. In addition, most of numerical studies on supersonic/ hypersonic boundary layer flow over the cones take the assumption of axial symmetric mean flow. In practice, however, the mean flows around the cones are not axial symmetric because of the nonzero angle of attack, and the
TABLE I. Flow parameters.

\begin{tabular}{lccccc}
\hline \hline $\mathrm{Re}_{n}$ & $\mathrm{Ma}_{\infty}$ & $\begin{array}{c}\mathrm{AoA} \\
(\mathrm{deg})\end{array}$ & $\begin{array}{c}T_{w} \\
(\mathrm{~K})\end{array}$ & $\begin{array}{c}T_{\infty} \\
(\mathrm{K})\end{array}$ & $\begin{array}{c}\theta_{h} \\
(\mathrm{deg})\end{array}$ \\
\hline 10000 & 6 & 1 & 294 & 79 & 5 \\
\hline \hline
\end{tabular}

boundary layer transition is strongly affected by this axial asymmetry. Experimental researches show that even a very small angle of attack (such as $1^{\circ}$ ) can affect the transition locations of cone boundary layer significantly. ${ }^{8,12}$ The authors ${ }^{13}$ performed the DNS of boundary transition over a blunt cone with free-stream Mach 6 and $1^{\circ}$ angle of attack, trying to answer the question why transition is early in the leeward section and is delayed in the windward section. Limited by computer resource, only the flow around the leeward and the windward sections is simulated in the DNS, ${ }^{13}$ while the whole distribution of transition locations on the cone surface is not studied. Stetson's experiment ${ }^{12}$ shows that the transition location shows a nonmonotonic curve on the cone surface in the condition of small angle of attack. However, the mechanism of this nonmonotonic transition location is still unclear and needs further study.

In the present study, the DNS of supersonic blunt cone boundary layer transition with free-stream Mach number 6 and $1^{\circ}$ angle of attack has been performed to study the mechanism of the transition. Different from the authors' previous work, ${ }^{13}$ fine meshes covers the region from the leeward to the windward section in the current DNS. Numerical result shows a nonmonotonic transition location profile on the cone surface. Transition onset location in $20^{\circ}-30^{\circ}$ planes is much delayed. The transition mechanism for different meridian planes is investigated.
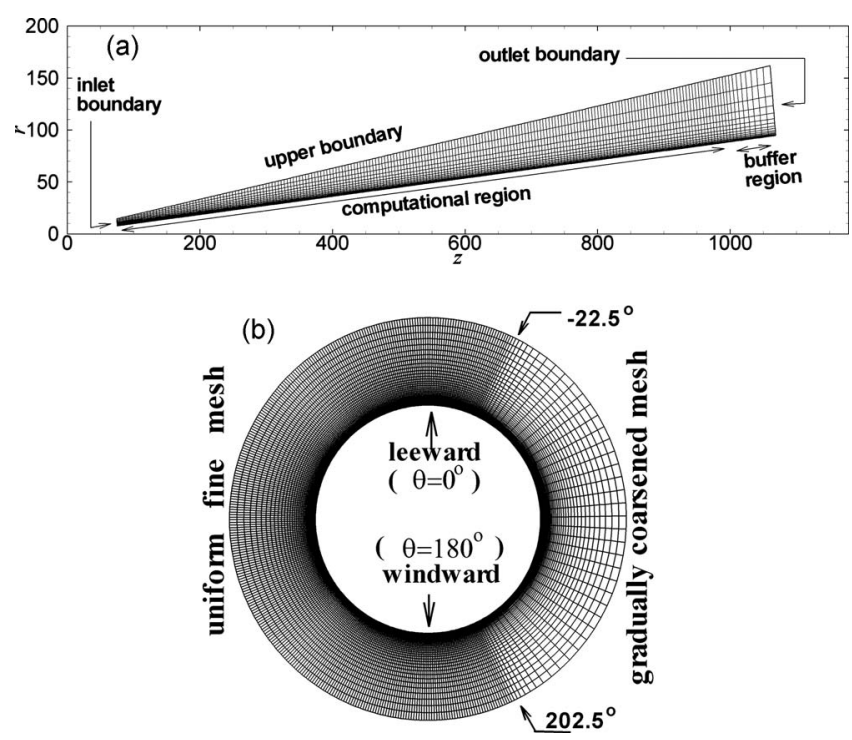

FIG. 2. Schematic of computational mesh. (a) Mesh in the axial section. (b) Mesh in the circumference section. 
TABLE II. Mesh parameters.

\begin{tabular}{ccccc}
\hline \hline $\begin{array}{c}\text { Computation } \\
\text { domain }\end{array}$ & $\begin{array}{c}\text { Mesh } \\
\text { number }\end{array}$ & $\Delta x^{+}$ & $\Delta y_{w}{ }^{+}$ & $r \Delta \theta^{+}(z=500)$ \\
\hline $75 \leq z \leq 1000,-22.5^{\circ} \leq \theta \leq 202.5^{\circ}$ & $2500 \times 90 \times 1400$ & 19.2 & 0.8 & 6.1 \\
\hline \hline
\end{tabular}

\section{NUMERICAL METHOD AND CODE VALIDATION}

\section{A. Numerical method}

Computational model for hypersonic flow over a blunt cone is shown in Fig. 1, and flow parameters are shown in Table I, where $\operatorname{Re}_{n}=\rho_{\infty} r_{n} u_{\infty} / \mu_{\infty}$ is the Reynolds number based on the cone's head radius $r_{n}$ and free-stream parameters. In our study, $r_{n}=1 \mathrm{~mm}$. $\mathrm{Ma}_{\infty}$ is the free-stream Mach number; AoA is the angle of attack; $T_{w}$ is the wall temperature; $T_{\infty}$ is the free-stream temperature; $\theta_{h}$ is the half cone angle. In this paper, all length variables are normalized by $r_{n}$, unless mentioned elsewhere.

Computations contain two steps. In the first step, steady flow is computed with the computational domain covering the cone's head and the bow shock. In the second step (unsteady simulation), the transition flow in the boundary layer is computed with the computational domain inside the bow shock that does not contain the cone's head (see Figs. 1 and 2). A parallel CFD software HOAM-OPENCFD developed by the authors are used in this DNS. Jacobian transformed compressible Navier-Stokes equations in cylinder coordinate are used and solved numerically by using high-order finite difference methods. Convection terms are split by using StagerWariming splitting and are discretized with seventh order WENO (weighted essentially non-oscillatory) scheme, ${ }^{14}$ whereas viscous terms are discretized with eighth order center finite difference scheme. Third order TVD (total variation diminishing)-type Runge-Kutta method ${ }^{14}$ is used for time advance.

Computational mesh of unsteady (transition) simulation is shown in Fig. 2. The computational domain is located inside the bow shock (see Fig. 1). The upper boundary in axial section is a line with $8.5^{\circ}$ tile angle to $z$-axial, and the computational domain in wall-normal direction contains more than ten times of boundary layer's thickness. Along streamwise direction, uniform fine mesh is used in the region $75 \leq z \leq 1000$, and gradually coarsened mesh is used in a buffer region $z>1000$ to minimize the reflection of outlet boundary.

The circumference computational domain covers the entire circumference $0^{\circ} \leq \theta \leq 360^{\circ}$. The uniform fine mesh covers a $225^{\circ}$ region $-22.5^{\circ} \leq \theta \leq 202.5^{\circ}$, and gradually coarsened mesh is used in the rest interval (see Fig. 2). It is helpful to minimize the computational cost that fine mesh covers the $225^{\circ}$ region rather than entirely $360^{\circ}$ region. Since statistics quantities are symmetrical in the leeward and the windward section, the flow in the region $0^{\circ} \leq \theta \leq 180^{\circ}$ is statistically equivalent to that in the region $180^{\circ} \leq \theta \leq 360^{\circ}$, and transition mechanism in the region $180^{\circ} \leq \theta \leq 360^{\circ}$ is the same as that in $0^{\circ} \leq \theta \leq 180^{\circ}$. However, we cannot simply set the computational domain to be $0^{\circ} \leq \theta \leq 180^{\circ}$ with the symmetry boundary in $\theta=0^{\circ}$ and $180^{\circ}$, because the tur- bulent fluctuations are not symmetrical in these two boundaries. Uniform fine mesh in the region $0^{\circ} \leq \theta \leq 360^{\circ}$ is also a choice, but this seems somewhat a wasting of computational resource, since we need not study the transition mechanism in the region $180^{\circ} \leq \theta \leq 360^{\circ}$. So, as an alterative, we use the computational domain cover $0^{\circ} \leq \theta$ $\leq 360^{\circ}$ and fine mesh cover $-22.5^{\circ} \leq \theta \leq 202.5^{\circ}$. Based on the results of the authors' previous research, ${ }^{13}$ perturbation waves propagate mainly along streamwise direction, and spanwise propagations are very weak. In the authors' previous research, ${ }^{13}$ the fine meshes are set in a $45^{\circ}$ range $-22.5^{\circ} \leq \theta \leq 22.5^{\circ}$ and gradually coarsened mesh is used in the rest interval. Numerical results of Ref. 13 show that the coarse mesh in the region $22.5^{\circ} \leq \theta \leq 337.5^{\circ}$ does not decay the transition and turbulence around the leeward section $\left(\theta=0^{\circ}\right)$. Based on this analysis, in the current simulation, the gradually coarsened mesh in the region $202.5^{\circ} \leq \theta \leq 337.5^{\circ}$ should have little effects on the results of the interested region $0^{\circ} \leq \theta \leq 180^{\circ}$. Numerical results of the current study (see Figs. 6, 8, and 10) also confirm it.

The computational domain, mesh number, and mesh resolution (in wall unit) are shown in Table II, where $z$ is the coordination in axial direction (see Fig. 1) and the length is normalized by the cone's head radius. The uniform streamwise grid space is $\Delta x^{+} \approx 19.2$ (if not specific, wall unit is measured by using the turbulent parameters at $z=800$ in the leeward section). The wall-normal grid space for the first grid to the wall is unchanged in the whole domain, and $\Delta y_{w}{ }^{+} \approx 0.8$. The wall-normal mesh is gradually coarsened to approximately $\Delta y^{+} \approx 10-15$ at the boundary layer's upper edge. Due to the geometry, spanwise grid space increases gradually along the streamwise direction. The nearwall spanwise grid space is $\Delta z^{+}=r \Delta \theta^{+} \approx 6.1$ in the region $z=500$, and the space is $\Delta z^{+} \approx 9.8$ in the region $z=800$.

Boundary conditions for simulations of unsteady flow are the same as those used in Ref. 13.

(1) Inflow boundary and upper boundary (see Fig. 2): time-

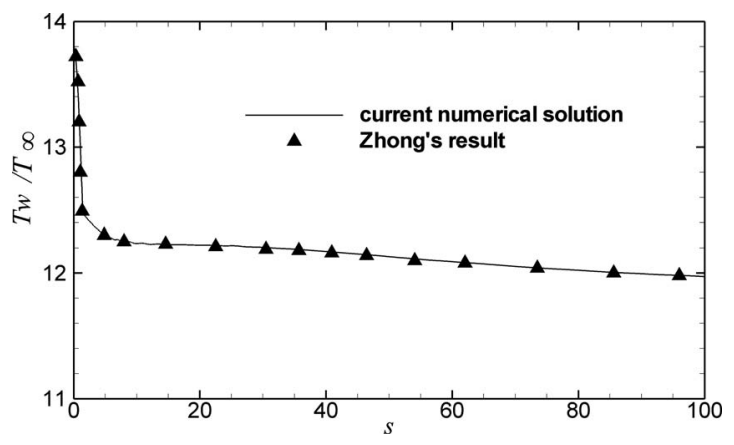

FIG. 3. Steady wall temperature compared with Zhong's result. 


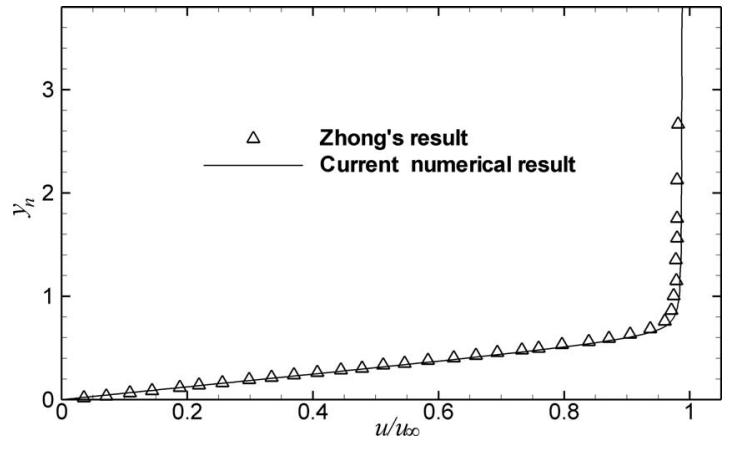

FIG. 4. Tangential velocity profile along the wall-normal direction at $s=94$ compared with Zhong's result.

independent conditions obtained from steady simulation of the first step.

(2) Nonreflecting boundary condition is implemented on the outflow boundary in the streamwise direction.

(3) Periodical boundary is used in circumference direction $\left(\theta=0^{\circ}\right.$ and $\left.\theta=360^{\circ}\right)$.

(4) Wall boundary: isothermal wall temperature together with assumption $\partial p / \partial y_{n}=0$ used on the wall. A second
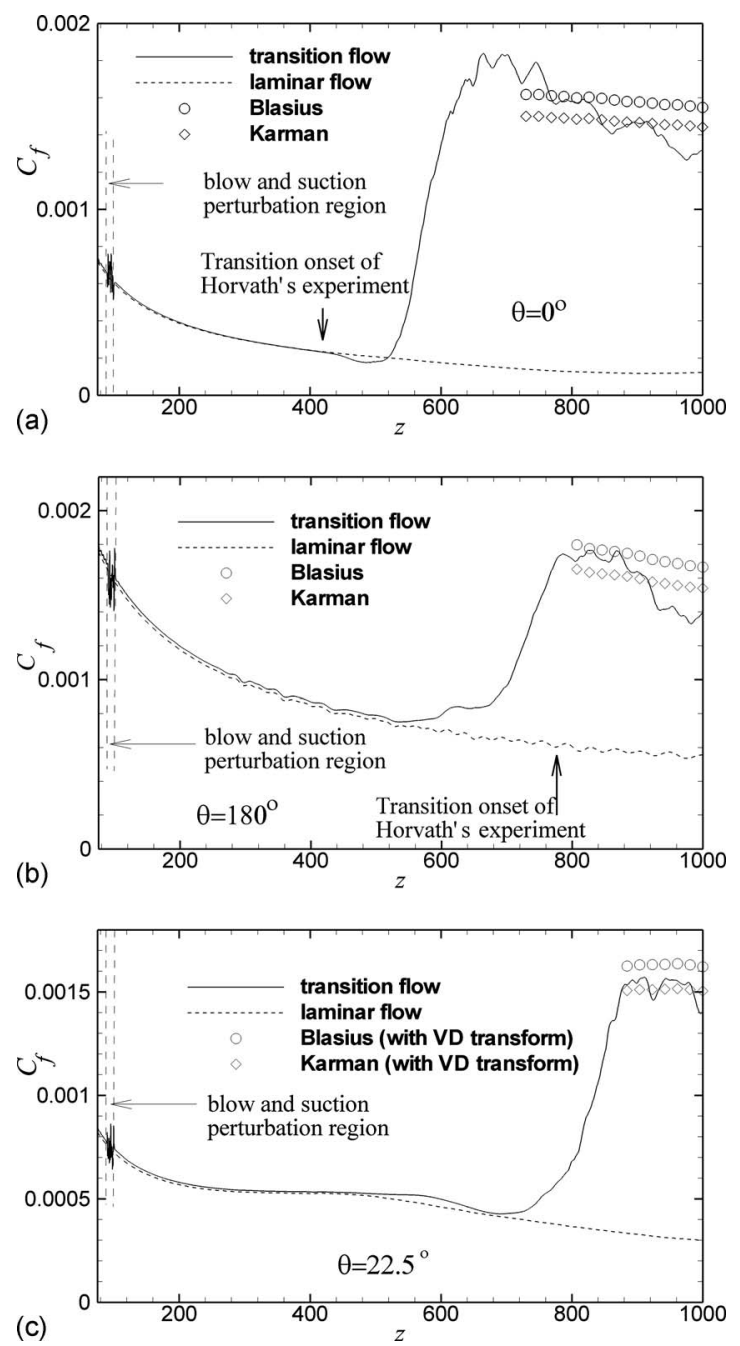

FIG. 5. Plot of fraction coefficient $C_{f}$ in the (a) leeward section, in the (b) windward section, and in the (c) section of $\theta=22.5^{\circ}$.
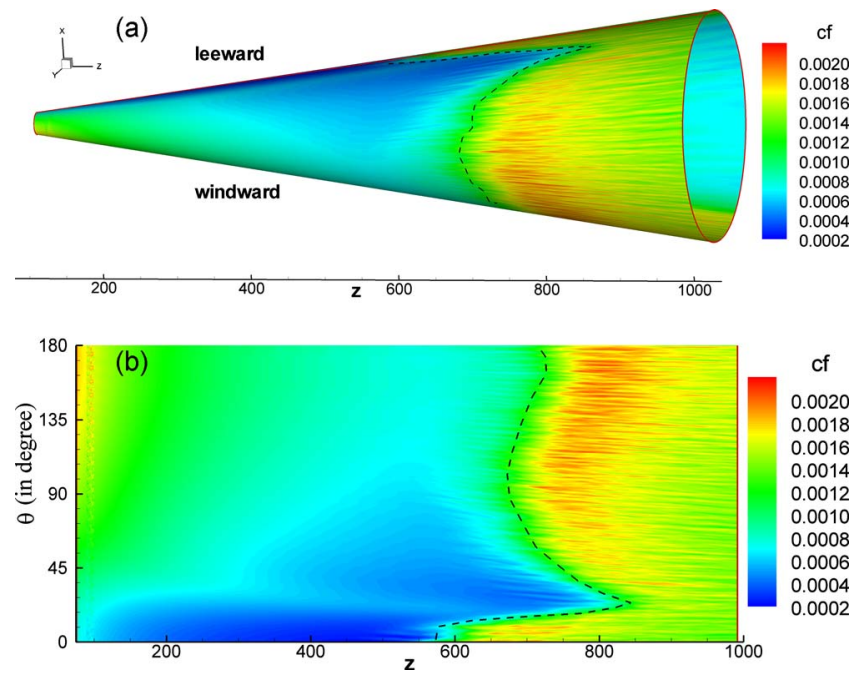

FIG. 6. (Color online) Distribution of skin fraction coefficient $C_{f}$ on the surface of the cone. The dashed lines indicate the rapid increasing location of $C_{f}$. (a) Contour in $C_{f}$ three-dimensional space. (b) Contour in $z-\theta$ plane $\left(\theta=0^{\circ}\right.$ denotes the leeward section, $\theta=180^{\circ}$ denotes the windward section).

order one-side finite difference scheme is used to compute the wall pressure. Wall blow and suction perturbation are used to trigger the transition. The streamwise and circumference velocities are taken as zero on the wall, and the wall-normal velocity on the wall is

$v_{n}= \begin{cases}v_{b s}, & z_{a} \leq z \leq z_{b} \\ 0, & \text { otherwise }\end{cases}$

where $v_{b s}$ is the wall blow-and-suction perturbation velocity imposed on the wall at the range $90=z_{a} \leq z \leq z_{b}$ $=100$. In this research, $v_{b s}$ is set to be random numbers between $-\varepsilon$ and $\varepsilon$, where $\varepsilon=0.01$.

Since nonreflecting or absorbing boundary conditions are not used at the upper boundary, there are numerical fluctuation reflections on this boundary. However, because the upper boundary is far enough from the boundary layer (more than ten times of boundary layer thickness), the numerical disturbances reflected from the upper boundary are very weak in this case. Moreover, as addressed by Adams, ${ }^{15}$ the mesh coarsening toward the upper boundary lets the numerical diffusion of the discretization scheme act as a filter that removes spurious wave reflections from the boundary. In our DNS, the numerical dissipation on the very large grid space near the upper boundary absorbs the reflection waves. In Ref. 13 , the authors tested the reflection waves from the upper boundary, and found that the fluctuation near the upper boundary layer is very small and the computational domain is not contaminated by nonphysical reflection waves from the upper boundary.

DNS is performed on supercomputers DeepComp 6800 in Supercomputing Center, Chinese Academy of Sciences, and Dawning 4000A in Shanghai Supercomputing Center. 256 CPU cores are used for the DNS cases. After long time computing, the flow becomes statistically steady, and 425 three-dimensional fields that span 1530 nondimen- 

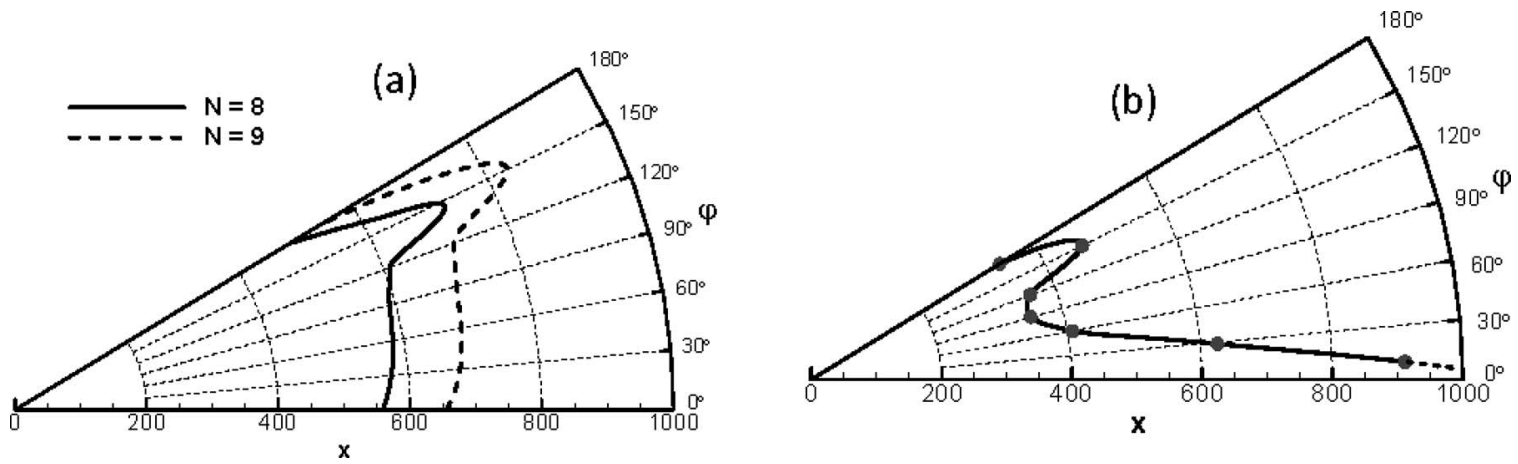

FIG. 7. Transition locations predicted by Su et al. (Ref. 10) (a) by using the conventional $e^{N}$ method and (b) by using the modified $e^{N}$ method. ( $x$ denotes the axial coordinate; $180^{\circ}$ denotes the leeward section; $0^{\circ}$ denotes the windward section in this figure.)

sional time units are used for time averaging. The total computation takes about $4000 \mathrm{~h}$ (about $1 \times 10^{6} \mathrm{CPU}$ hours) for the whole computation.

\section{B. Code validation}

In order to validate the code, we simulate the steady flow of Mach 7.99 flow over a blunt cone with zero angle of attack. ${ }^{9}$ The flow conditions are free-stream Mach number $\mathrm{Ma}_{\infty}=7.99$, Reynolds number $\operatorname{Re}_{n}=\rho_{\infty} u_{\infty} r_{n} / \mu_{\infty}=33449$, half-cone angle $\theta_{\eta}=7^{\circ}$, adiabatic wall, and free-stream temperature $54.474 \mathrm{~K}$. Zhong et al. ${ }^{9}$ give a high quality numerical solution of this steady boundary layer flow by using their high order shock-fit method. To test our code, we compare our result with that of Zhong et al. Our steady numerical simulation is carried out by using the current code and in a mesh with 1600 (streamwise) $\times 160$ (wall normal) $\times 8$ (circumference) grid points. Since the attack of angle is zero, the steady flow is two dimensional (axisymmetrical), and the mesh number in circumference does not affect the numerical accuracy. Figure 3 gives wall temperature distributions along the cone surface, and Fig. 4 gives the tangential velocity profile along the wall-normal direction at $s=94$. Those two figures show a very good agreement between our numerical results and Zhong's results, which validates our code.

Additionally, the current code (software "HOAMOPENCFD") has been validated through DNS cases of turbulent/transition flows of flat-plate boundary layer, sharpcone boundary layer, and blunt-wedge boundary layer. Details can be found in the authors' papers. ${ }^{13,16-18}$

\section{FLOW STATISTICS AND VISUALIZATION}

Figures 5(a)-5(c) show the skin fraction coefficient $C_{f}$ along the streamwise direction in the leeward section
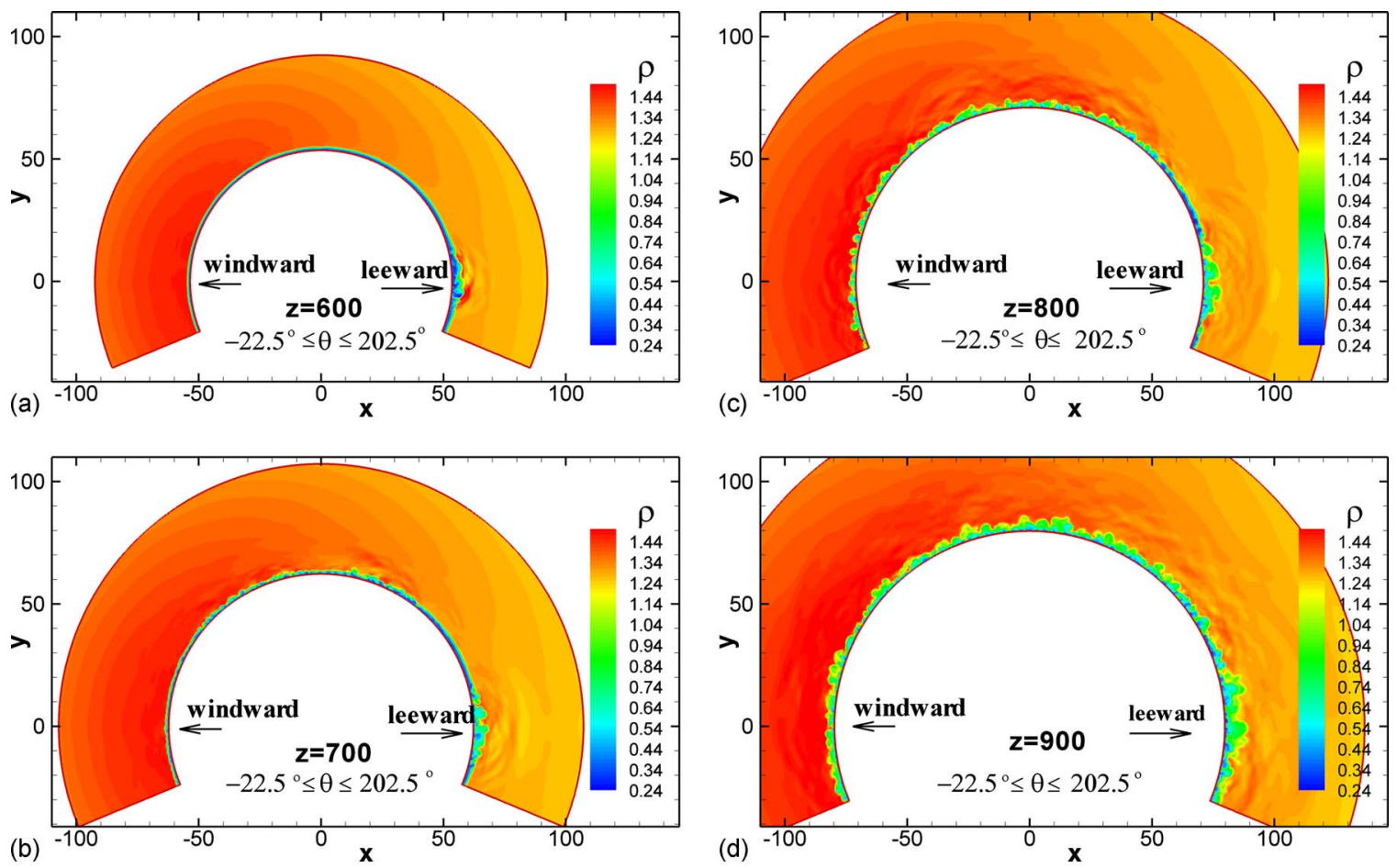

FIG. 8. (Color online) Contours of instantaneous density at three wall-normal planes with $z=600,700,800$, and 900. 

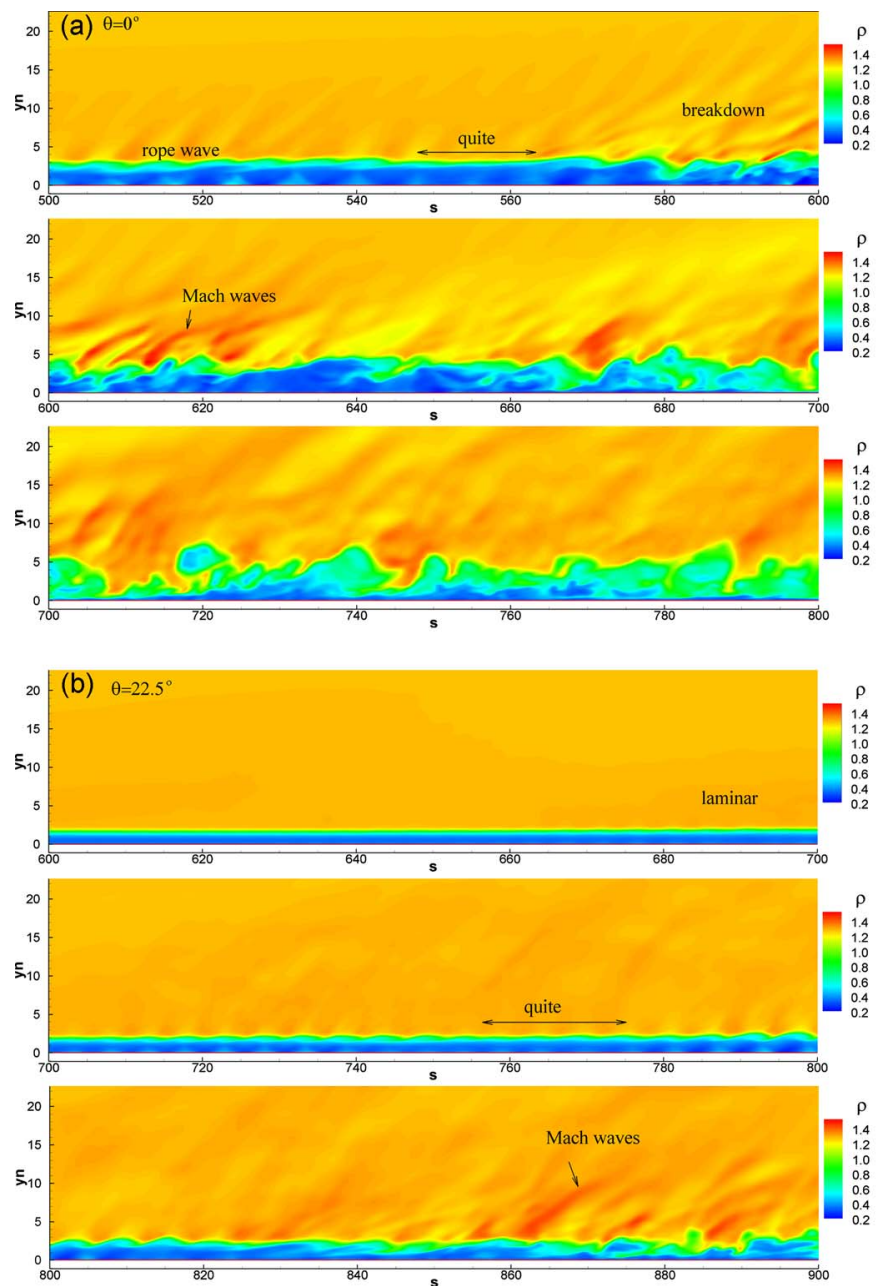
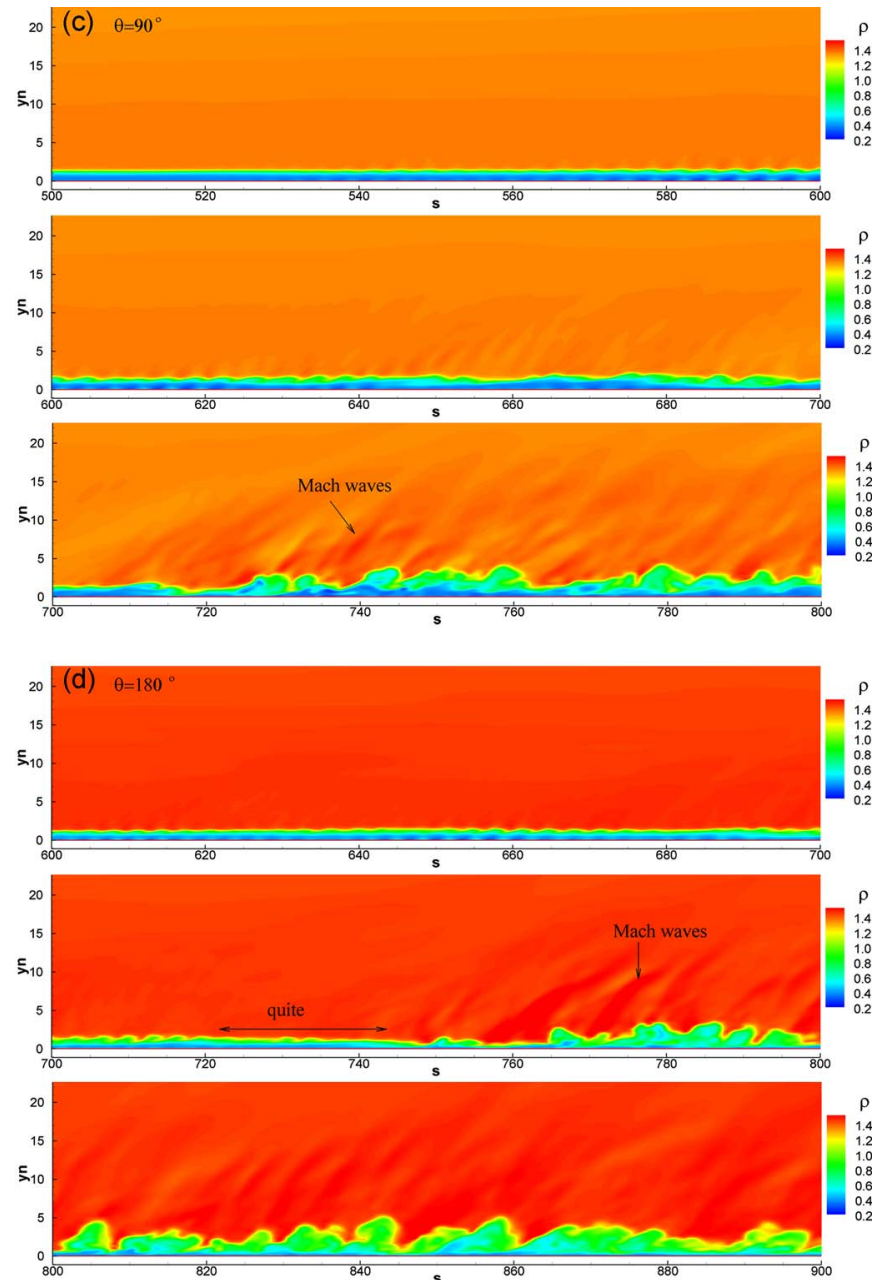

FIG. 9. (Color online) Contours of instantaneous density at four meridian planes with (a) $\theta=0^{\circ}$ (leeward section), (b) $\theta=22.5^{\circ}$, (c) $\theta=90^{\circ}$, and (d) $\theta=180^{\circ}$ (windward section).

$\left(\theta=0^{\circ}\right)$, the windward section $\left(\theta=180^{\circ}\right)$, and the $\theta=22.5^{\circ}$ section, respectively, where

$$
\begin{aligned}
& C_{f}=\frac{\tau_{w}}{1 / 2 \rho_{\infty} u_{\infty}^{2},} \\
& \tau_{w}=\left.\bar{\mu}_{w} \frac{\partial \bar{u}_{s}}{\partial y_{n}}\right|_{w},
\end{aligned}
$$

$\bar{u}_{s}$ is the time-averaged streamwise velocity, and $y_{n}$ is the distance to the wall.

The solid line denotes the result of the current DNS and the dashed line denotes the result of the laminar flow. The horizontal coordinate $z$ denotes axial location (see Fig. 1) normalized by the nose radius. There is still no strict definition of transition location of boundary layer flow, but we can estimate the transition location approximately from the growth of $C_{f}$. The location where the $C_{f}$ line begins to grow rapidly is often considered as the transition (onset) location. According to this criterion, transition occurs at $z \approx 500$ $\left(\operatorname{Re}_{z} \approx 5 \times 10^{6}\right)$ in the leeward section, occurs at $z \approx 700$ $\left(\operatorname{Re}_{z} \approx 7 \times 10^{6}\right)$ in the windward section, and occurs at $z \approx 750\left(\operatorname{Re}_{z} \approx 7.5 \times 10^{6}\right)$ in the section $\theta=22.5^{\circ}$. In Fig. 5, the theoretical values of turbulent skin friction coefficients are also plotted, and it is shown that our numerical result agrees well with the theoretical result. Here the theoretical values are computed through the momentum thickness by using Karman-Schoenherr and Blasius formulations with Van Driest II transformation (see Ref. 19 for details of the theoretical formulas).

Horvath et al. ${ }^{8}$ reported the experimental results for transition locations of Mach 6 flow over cones and flared cones with $5^{\circ}$ half-cone angle and different bluntness, different angle of attacks, and different Reynolds numbers. Horvath's report includes the data obtained from the NASA LaRC Mach 6 low turbulence tunnel. Transition locations obtained in low turbulence tunnels are closer to that of actual (flight) situations than that obtained in convention tunnels, therefore transition locations obtained in low turbulence tunnels are more reliable for the design of hypersonic vehicles. From Horvath's experimental results, it can be deduced that the transition onset Reynolds numbers should be $\operatorname{Re} \approx 4.2 \times 10^{6}$ in the leeward section and $\operatorname{Re} \approx 7.8 \times 10^{6}$ in the windward section for the quiet tunnel test of a blunt cone with the same half-cone angle (5 degree), the same angle of attack (1 degree), the same free-stream Mach number $(\mathrm{Ma}=6)$, and approximately the same bluntness as these in our numerical 

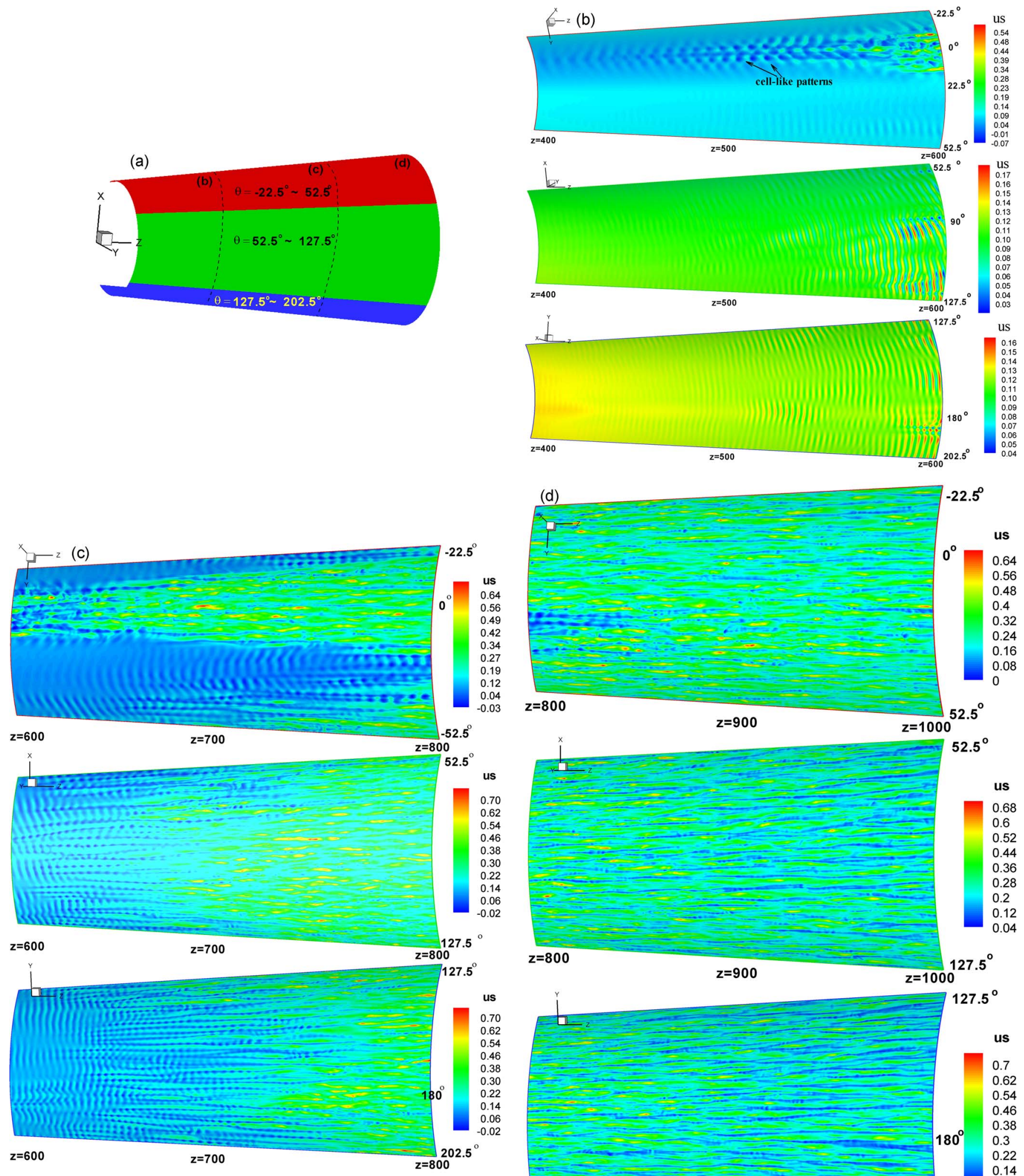

(d)
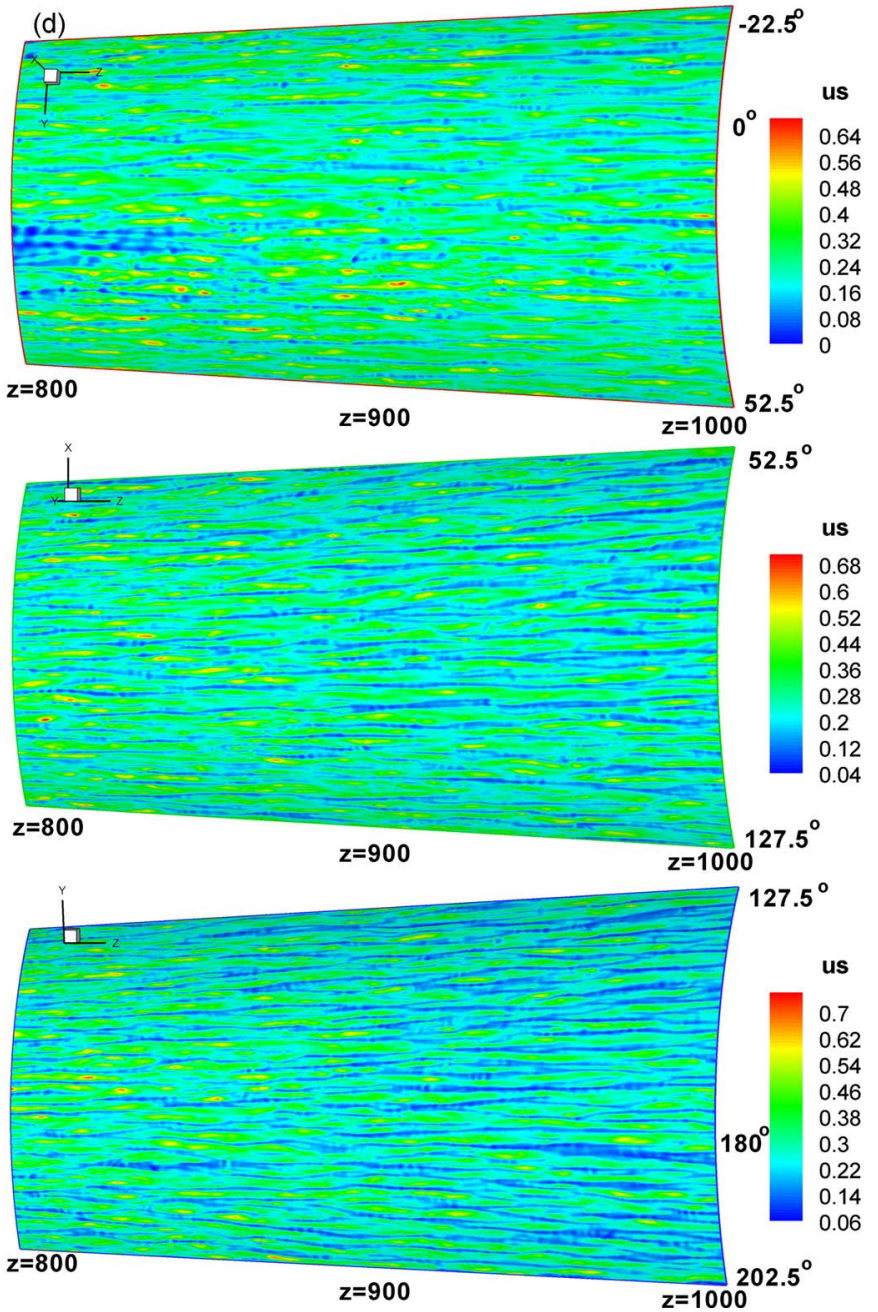

FIG. 10. (Color online) Contours of instantaneous streamwise velocity $u_{\mathrm{S}}$ in a wall-parallel curved face with wall distance $y_{n} \approx 0.1\left(y_{n}^{+} \approx 5\right)$. (a) Schematic of visualization domain; visualization domain: (b) $400 \leq z \leq 600$, (c) $600 \leq z \leq 800$, and (d) $800 \leq z \leq 1000$. 
TABLE III. Growth rates $\left(-\alpha_{i}\right)$ of the most unstable second modes in different locations.

\begin{tabular}{llcc}
\hline \hline$z$ & $\theta=0^{\circ}$ & $\theta=180^{\circ}$ & $\theta=22.5^{\circ}$ \\
\hline 100 & 0.042 & 0.057 & 0.030 \\
200 & 0.052 & 0.056 & 0.039 \\
300 & 0.047 & 0.051 & 0.033 \\
400 & 0.043 & 0.048 & 0.028 \\
500 & 0.040 & 0.043 & 0.033 \\
600 & 0.038 & 0.041 & 0.037 \\
\hline
\end{tabular}

simulation. Our numerical result agrees approximately with the result of the quiet tunnel test of Horvath et al. ${ }^{8}$

Figure 6 shows the distribution of skin fraction coefficient $C_{f}$ on the surface of the cone. Figure 6(a) is the threedimensional visualization on cone surface and Fig. 6(b) is the two-dimensional contour in the $z-\theta$ plane. The dashed lines in both figures indicate the rapid increasing region of $C_{f}$, which mark the transition locations on the cone surface. The dashed lines indicate nonmonotonic transition location layout on the cone surface and the transition locations are delayed significantly in the range $20^{\circ} \leq \theta \leq 30^{\circ}$. The transition location in the section of $\theta=22.5^{\circ}$ is the most retarded section in the whole cone surface.

Su et al. ${ }^{10}$ computed the transition location on the cone surface by using the conventional $e^{N}$ method and their modified $e^{N}$ method based on the laminar flow profiles in the same flow conditions of the current research. The transition locations of the computation of Su et al. ${ }^{10}$ are shown in Fig. 7. In this figure, symbol $x$ denotes axial-direction coordinate (as $z$ in this paper) and symbol $\varphi$ denotes circumference angle. As defined in the computation of Su et al. ${ }^{10} \varphi=0^{\circ}$ denotes the windward section (as $\theta=180^{\circ}$ in this paper) and $\varphi=180^{\circ}$ denotes the leeward section. Figure 7 shows that transition location is delayed in the section $\varphi \approx 150^{\circ}\left(\theta \approx 30^{\circ}\right)$ and this agrees with our numerical result approximately.

Contours of instantaneous density in four wall-normal planes (the planes normal to the generating line of the cone) with $z=600,700,800$, and 900 are shown in Fig. 8. This figure shows that transition has occurred around the leeward section $\left(-10^{\circ} \leq \theta \leq 10^{\circ}\right)$, whereas the rest range keeps laminar at $z=600$. While at $z=800$, flows in all $\theta$-range except

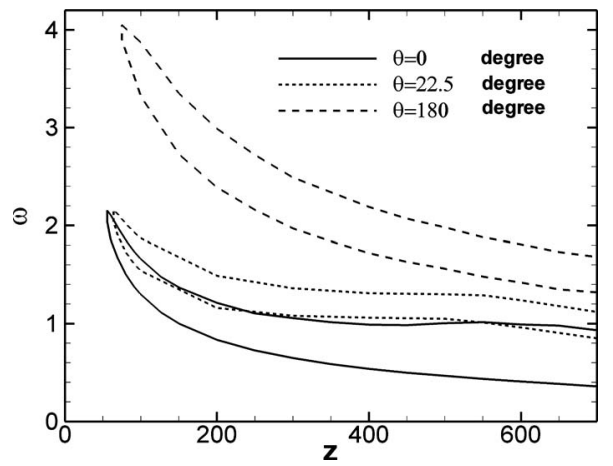

FIG. 11. Neutral stability curves in $0^{\circ}$ (leeward), $22.5^{\circ}$, and $180^{\circ}$ (windward) sections.

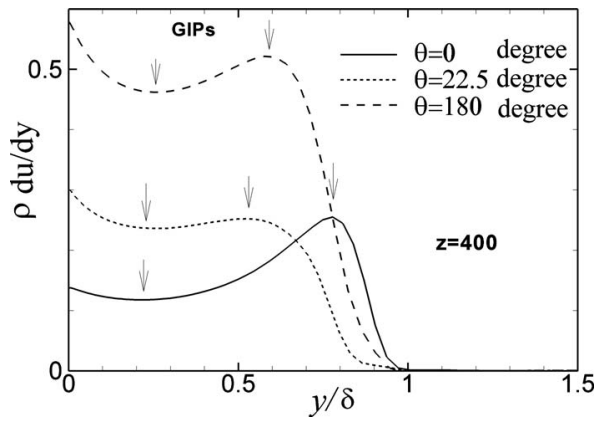

FIG. 12. Profiles of $\rho d u / d y$ in $z=400$ with $\theta=0^{\circ}, 22.5^{\circ}$, and $180^{\circ}$. Arrows mark the GIPs.

$20^{\circ} \leq \theta \leq 30^{\circ}$ become turbulent, and the range $20^{\circ} \leq \theta \leq 30^{\circ}$ is the latest transition range. This figure also shows highly intermittent characters of the outer layer of the boundary layer and Mach waves radiated from the boundary layer's outer edge.

Contours of instantaneous density in four meridian planes with $\theta=0^{\circ}, 22.5^{\circ}, 90^{\circ}$, and $180^{\circ}$ are shown in Figs. 9(a)-9(d). Figure 9(a) shows rope waves at the edge of boundary in the leeward section $\left(\theta=0^{\circ}\right)$ within the range $500<z<580$, which is the feature of the second Mack mode waves. In the range $z>580$, the edge of density boundary layer becomes irregular and strongly disturbed, and the boundary layer breakdown occurs. It can be seen that the radiation of strong Mach waves propagates from the edge of boundary layer during the transition stage. The rope waves and the Mach wave radiation are also found in Figs. 9(b)-9(d). Figure 9(b) shows contours of density in the section $\theta=22.5^{\circ}$. It can be found that the transition location is much delayed compared with that in the leeward section [Fig. 9(a)]. The density distribution keeps laminar in the range $z<700$ and disturbance waves become observable in this figure in the range $z>700$. Figures 9(a)-9(d) show that the boundary layer becomes relatively quiet just before the breakdown stage, and the mechanism should be further studied.

Figure 10 shows the instantaneous streamwise velocity in a curved plane parallel to the wall with the wall distance $y_{n} \approx 0.1\left(y_{n}^{+} \approx 5\right)$. The total visualization domain is $-22.5^{\circ}$ $<\theta<202.5^{\circ}, 400<z<1000$. Figure 10 (b) shows that there are cell-like patterns upstream the transition range $(450<z$ $<570)$ around the leeward section $\left(\theta=0^{\circ}\right)$, and there are quasi-two-dimensional waves around the windward section $\left(\theta=180^{\circ}\right)$. From Figs. 10(b) and 10(c), it can be seen that the cell-like patterns break down to streamwise streaklike struc-

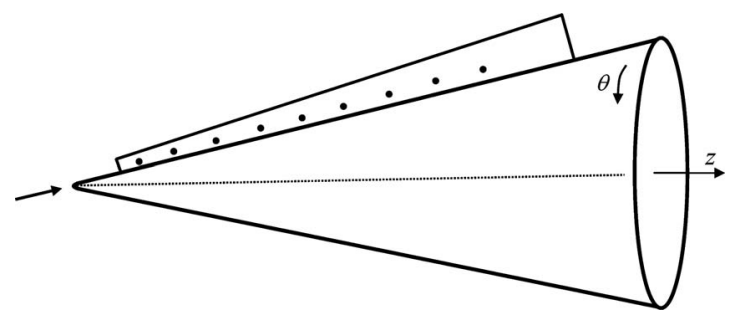

FIG. 13. Schematic of measuring points in the flow field. 

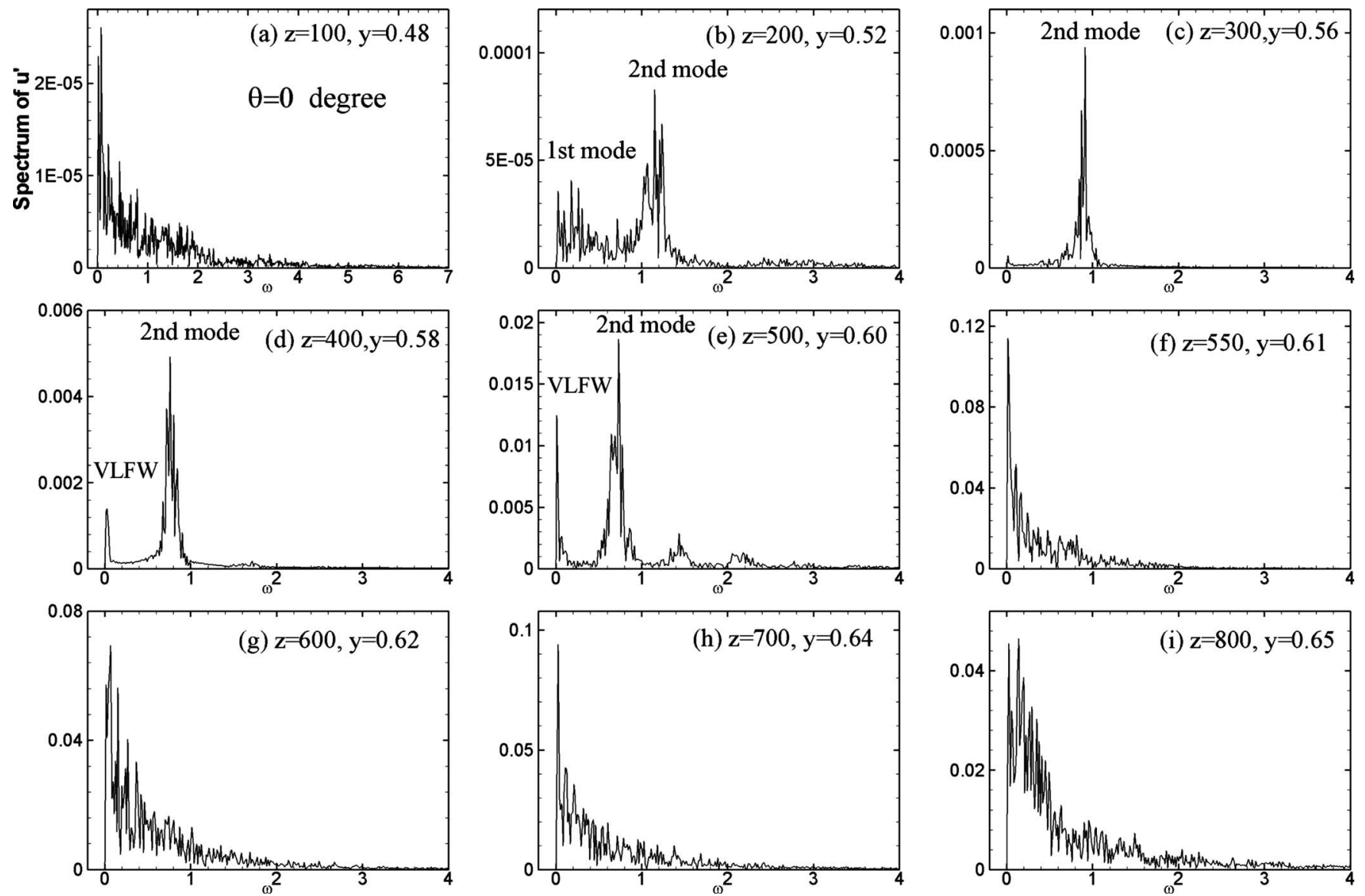

FIG. 14. Spectra of streamwise velocity fluctuations in the $\theta=0^{\circ}$ section.

tures in the range $570<z<650$ around the leeward section. Figure 10(c) shows that distortions of the two-dimensional waves occur around the windward section $\left(\theta=180^{\circ}\right)$ in the range $580<z<700$, followed by the formation of streamwise streaklike structures.

\section{LINEAR STABILITY THEORY ANALYSIS}

To study the mechanism why transition in the range $20^{\circ} \leq \theta \leq 30^{\circ}$ is delayed, both linear stability theory (LST) analysis and frequency spectrum are performed. LST based on the mean profiles obtained from the three-dimensional steady numerical solution is conducted to study the disturbance waves. According to LST, disturbance waves have the expression

$$
\mathbf{f}^{\prime}=\hat{\mathbf{f}}\left(y_{n}\right) e^{i\left(\alpha s+\beta z^{\prime}-\omega t\right)},
$$

where $\mathbf{f}^{\prime}=\left[\rho^{\prime}, u^{\prime}, v^{\prime}, w^{\prime}, T^{\prime}\right]^{T}$ is the disturbance and $\hat{\mathbf{f}}\left(y_{n}\right)$ is its eigenfunction. $\alpha, \beta$, and $\omega$ are streamwise wave number, spanwise wave number, and angular frequency of disturbance waves, respectively. $z^{\prime}$ is a local spanwise coordinate and $s$ is a cone surface distance from the leading. $-\alpha_{i}=-\operatorname{Im}(\alpha)$ is the growth rate of the disturbance wave and $-\alpha_{i}>0$ means unstable wave. In our LST analysis, a finite difference code SAYR is used (courtesy of Professors Zhou and Luo of Tianjin University). By search in the parameter plane of $(\omega, \beta)$, we can find the most unstable first and second Mack modes. The growth rate of the second mode is usually much higher than that of the first modes, which indicates that the second mode dominates the transition. ${ }^{13}$ The growth rates $\left(-\alpha_{i}\right)$ of the most unstable second modes in three meridian planes are given in Table III, where $z$ is the axial location normalized by the nose radius. This table shows that the second modes in the windward section $\left(\theta=180^{\circ}\right)$ are most unstable, but the transition location in the windward section is later than that in the leeward section. The delayed transition in the windward section cannot be interpreted simply by the lower growth rate of the unstable waves. The reason why transition is delayed in the windward section is studied by the authors ${ }^{13}$ by using joint frequency spectrum and LST analysis. It is found that the growth of

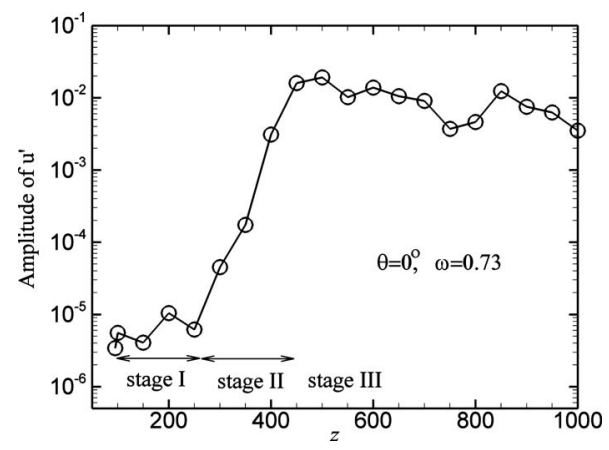

FIG. 15. Amplitude of perturbations wave $u^{\prime}$ in the leeward section with $\omega=0.73$. 


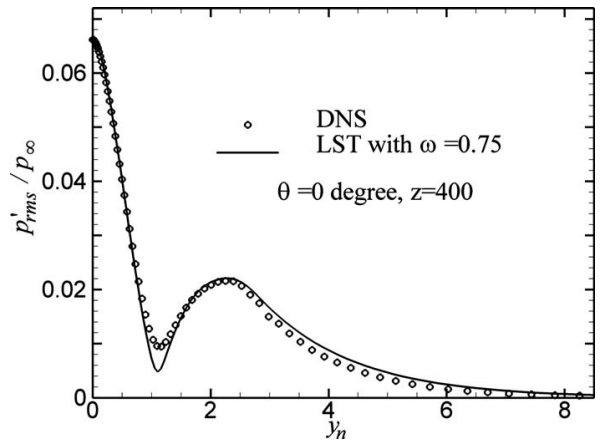

FIG. 16. Profiles of rms pressure fluctuation at a wall-normal line in the leeward section with $z=400$ compared with LST.

perturbation waves in the windward starts much later than that in the leeward section, and this results in the delayed transition in the windward section. In this paper, attentions are paid on the transition around the $\theta=22.5^{\circ}$ section. From Table III, it can be seen that the growth rate of the unstable second mode waves in the section $\theta=22.5^{\circ}$ is much lower than that in the leeward section and the windward section, which indicates that transition around the $\theta=22.5^{\circ}$ should be delayed.

Figure 11 shows the neutral stability curves of the second Mack disturbance waves in the section $\theta=0^{\circ}, 22.5^{\circ}$, and $180^{\circ}$, where $\omega$ denotes the angular frequency of the disturbance wave. Disturbance waves with the frequency inside the neutral curve are unstable. This figure shows that the unstable frequency range is much narrower in the section $\theta=22.5^{\circ}$, which is unfavorable for the growth of the perturbation waves here.

Figure 12 shows the one-dimensional plot of $\rho d u / d y_{n}$ in three meridian planes of $z=400$, where $u$ denotes the streamwise velocity and $y_{n}$ denotes the wall distance. The peak points of this plot are the GIPs of velocity, ${ }^{1}$ which are marked by the arrows in Fig. 12. According to the theory of Lees et al., ${ }^{1}$ to have a GIP in flow plot is a necessary condition of inviscousity instability. Figure 12 shows that all the three plots have GIPs, and this allows the flows in the leeward, windward, and $\theta=22.5^{\circ}$ sections to be inviscous unstable (or have the Mack second mode instability). Figure 12 shows that GIPs in the leeward and the windward sections are very clear, but the GIPs in the $\theta=22.5^{\circ}$ section are not so clear, and this denotes that the Mack second mode instability in this section is not as strong as the leeward section and the windward section. This agrees with the LST result in Table III.

\section{FREQUENCY SPECTRUM AND PERTURBATION WAVE ANALYSIS}

To further study the mechanism of transition, frequency spectrum analysis is performed. As shown in Fig. 13, several measuring points are set in the flow field in different meridian planes. Time series of flow variables (velocities, pressure,
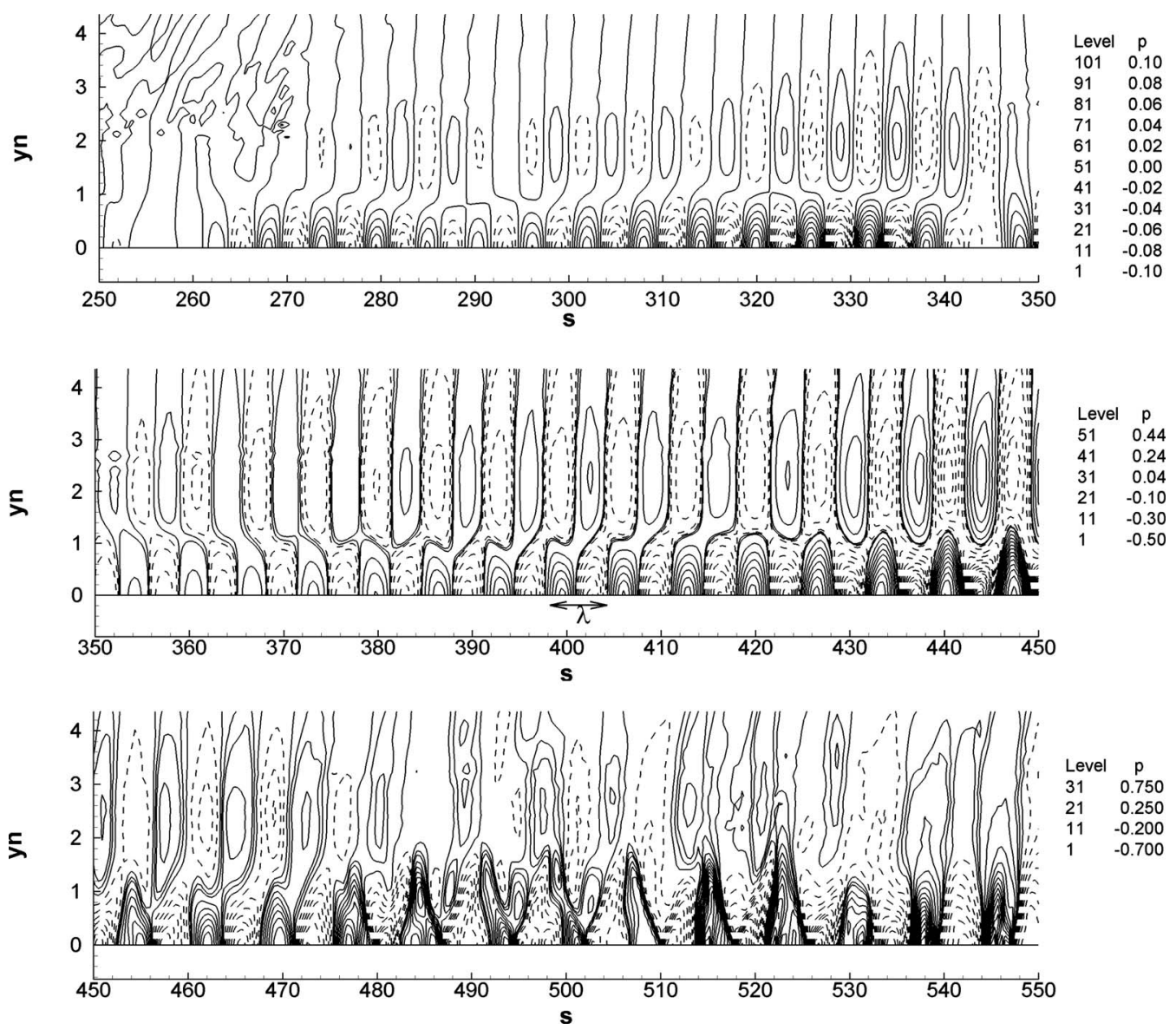

FIG. 17. Two-dimensional distribution of instantaneous pressure fluctuation in the leeward section: DNS data at $t=1799.64$. 


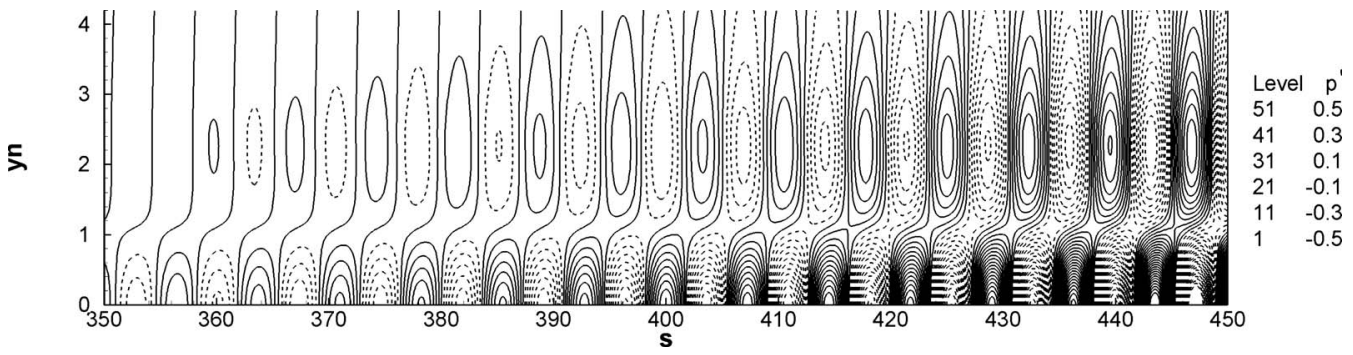

FIG. 18. Two-dimensional distribution of instantaneous pressure fluctuation in the leeward section: LST data of Mack second mode.

density, and temperature) in each point are recorded during the DNS, and frequency spectrum analysis is performed based on these time series. The time-advancing fluid data in three meridian planes with $\theta=0^{\circ}, 20^{\circ}$, and $180^{\circ}$ are also saved during the DNS, and totally 3747 two-dimensional fields are saved in the time span of 450 nondimensional time units. Perturbation waves in the three meridian planes are analyzed based on these saved data; frequency spectrum analysis, two-dimensional visualization, and band-pass filter analysis are used in the study.

\section{A. The leeward section $\left(\theta=0^{\circ}\right)$}

\section{Frequency spectrum analysis}

Figure 14 shows the spectra of streamwise velocity fluctuations at nine points in the leeward section. The location of these nine points is marked in this figure, and the distances to the wall of these points $\left(y_{n}\right)$ are approximately $0.6-0.7$ times of the local boundary layer's thickness. Streamwise velocity fluctuations recoded in these points contain effects of both the first and the second modes. Wideband spectrum is shown in the initial region $(z=100)$, which denotes the character of random disturbance of the wall blow and section. Two peaks are shown in the spectrum at $z=200$. One peak is located in the low frequency region $(\omega \leq 0.5)$, which is related with the disturbance waves of the first mode; the other peak locates in the higher frequency range $\omega=1.0-1.3$, which is related with the disturbance waves of the second mode. At $z=300$, the spectrum shows one peak in the frequency range $\omega=0.8-1.0$, which is located in the unstable frequency range of the second Mack mode (see the neutral stability curves in
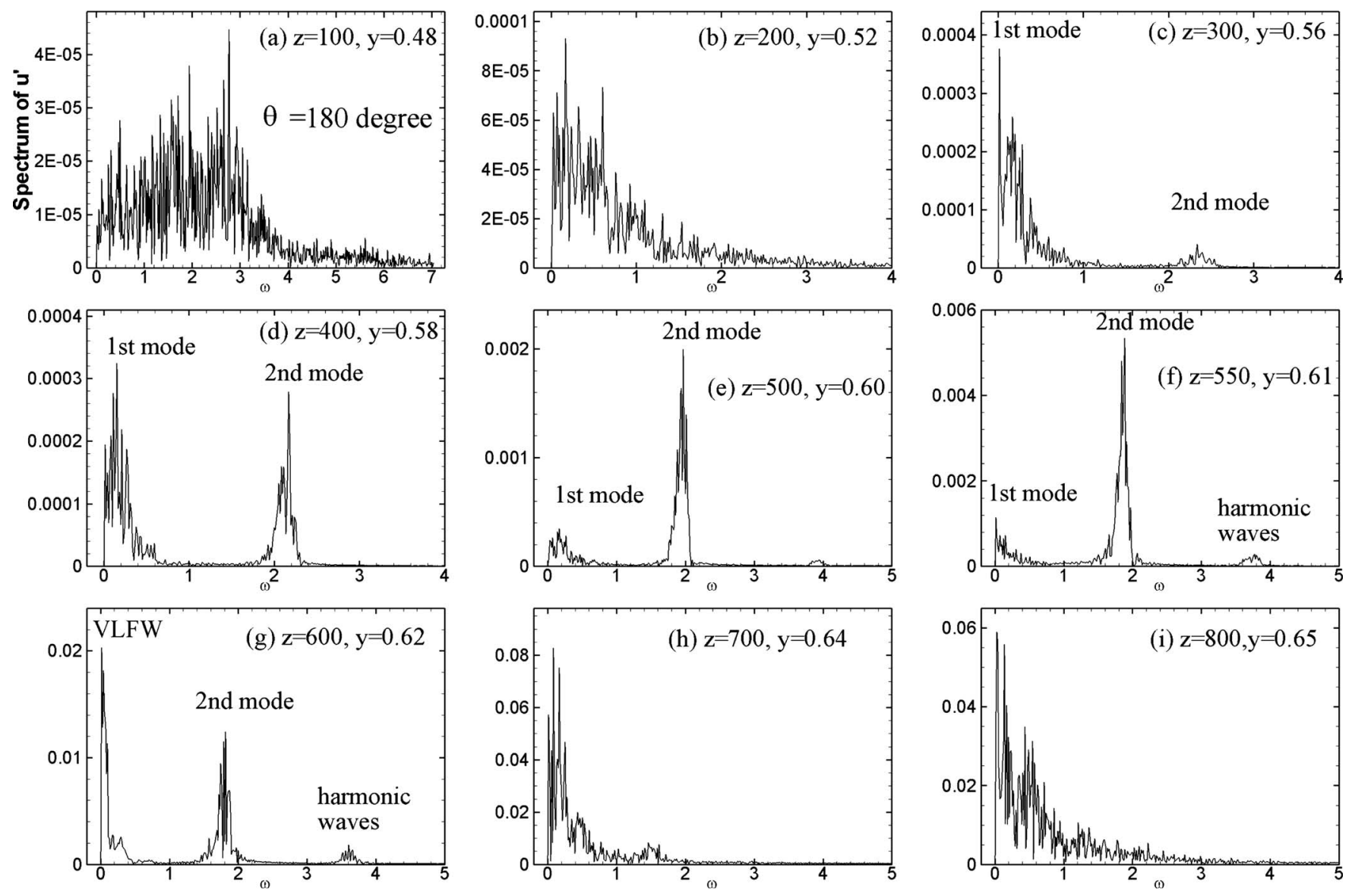

FIG. 19. Spectra of streamwise velocity fluctuations in the windward section $\left(\theta=180^{\circ}\right)$. 


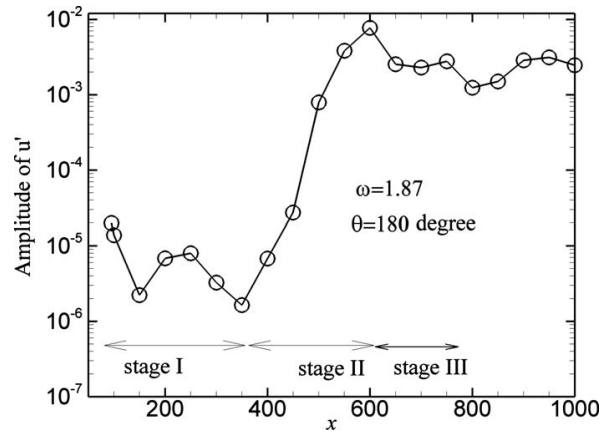

FIG. 20. Amplitude of perturbations wave $u^{\prime}$ in the windward section with $\omega=1.87$.

Fig. 11). At $z=400$, the spectrum shows two peaks. Besides the second Mack mode's peak $(\omega=0.75)$, there is a very low frequency peak with $\omega \approx 0.05$. This denotes that very low frequency wave (VLFW) occurs in the flow. At $z=500$, the second mode's peak with $\omega=0.73$ still dominates, but the VLFW's peak is more significant. Since the disturbance waves are strong enough here, VLFW should be some kind of nonlinear perturbations, and the characters of VLFW will be studied in Secs. V B 2 and V C 2.

Figure 15 shows the amplitude of streamwise perturbations wave $u^{\prime}$ with $\omega=0.73$ in the leeward section, and the wave with frequency $\omega=0.73$ is the dominate perturbation wave just before the transition [see Fig. 14(d)]. This figure shows three stages in the development history of this wave. Stage I $(z \leq 250)$ is the receptivity stage, and perturbation waves are excited by external perturbations (random blow and suction during $90 \leq z \leq 100)$ in this stage. Phenomena such as boundary-layer receptivity, mode chosen, and competition usually occur in this stage. Stage II $(250 \leq z \leq 450)$ is the linear growth stage, and this figure shows that the amplitude of the disturbance wave keeps nearly an exponential growth in this stage. The amplitude grows by 2580 times (approximately $e^{7.9}$ times) in the distance of 200 times of head radius (i.e., $200 \mathrm{~mm}$ ), and the mean growth rate is $-\bar{\alpha}_{i} \approx 0.04$, which is approximately close to the growth rate of LST prediction for the Mack second mode most unstable waves (see Table III).

Figure 16 shows the profile of rms pressure fluctuation $p_{\text {rms }}^{\prime}$ in a wall-normal line in the leeward section with $z=400$, and the result of the LST second mode is also plotted. This figure shows that the one-dimensional distribution of $p_{\text {rms }}^{\prime}$ is very close to the LST's prediction, and indicates that the second mode LST perturbation is the dominate perturbation here.

\section{Perturbation wave visualization}

Figure 17 shows the two-dimensional distribution of instantaneous disturbance pressure $p_{\mathrm{DNS}}^{\prime}=(p-\bar{p}) / p_{\infty}$ in the leeward section at $t=1799.64$ of the current DNS. The abscissa $s$ denotes the surface length from the leading, and according to the geometry of the $5^{\circ}$ blunt cone,

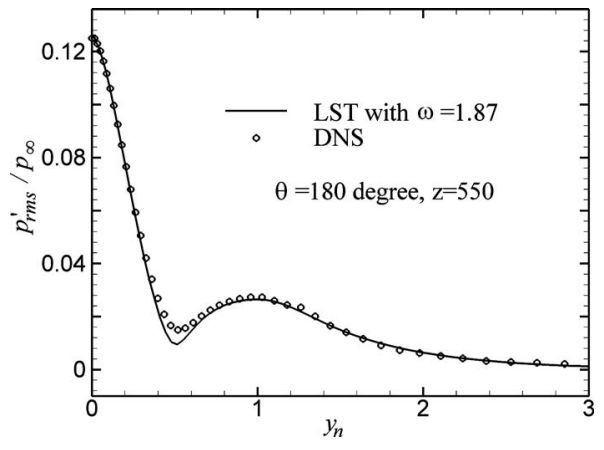

FIG. 21. Profiles of rms pressure fluctuation at a wall-normal line in the windward section with $z=550$ compared with LST.

$$
s=1.0038 z+0.0875,
$$

where $z$ is the axialwise distance (see Fig. 1). Since $s \approx z$, we do not strictly distinct them when the position need not a very accurate description. The ordinate $y_{n}$ denotes distance to the wall. This figure shows that a dominating sinuouslike disturbance wave is formed since $s \approx 260$ and grows stronger in the downstream range, and the disturbance wave is distorted after $s \approx 450$. The wavelength of this sinuouslike wave is $\lambda_{\mathrm{DNS}} \approx 7.0$ at the region $s \approx 400$ (marked in Fig. 17), which is close to the local second mode's wavelength $\left(\lambda_{\mathrm{LST}} \approx 7.2\right.$ with $\omega=0.75)$.

To further test the disturbance wave, we compare DNS result with the LST disturbance wave. Figure 18 plots the two-dimensional distribution of the second mode pressure disturbance wave with $\omega=0.75$, which is the frequency of the dominating peak in Fig. 14(d). The LST disturbance wave is

$$
p_{\mathrm{LST}}^{\prime}\left(s, y_{n}\right)=\hat{p}\left(y_{n}\right) \exp \left[i \alpha\left(s-s_{0}\right)\right],
$$

where $s_{0}=400 . \alpha$ and $\hat{p}$ are the eigenvalue and the eigenfunction of the LST mode, respectively, which are computed by using LST based on the mean profiles at $\left(s=400, \theta=0^{\circ}\right)$. According to LST solution for the second mode with $\omega=0.75, \alpha=0.87-0.025 i$. Compared with Fig. 17, we can see that the two-dimensional distribution of $p_{\mathrm{DNS}}^{\prime}$ is very close to that of $p_{\mathrm{LST}}^{\prime}$ in the region $360 \leq s \leq 440$, and the waves' scales (wavelength and wall-normal scale) are also very similar to each other. This shows that the second mode is the dominate wave in this region, and this also indicates that the second mode is the dominate perturbation wave to trigger the transition.

\section{B. The windward section $\left(\theta=180^{\circ}\right)$}

\section{Frequency spectrum analysis and disturbance wave visualization}

Figure 19 shows the spectra of streamwise velocity fluctuations in the windward section. This figure shows that at the early stage $(z<300)$ the low frequency disturbance (in the unstable frequency range of the first mode) dominates. However, a high frequency disturbance wave is amplified faster, and high frequency waves (in the unstable frequency range of the second mode) dominate downstream at $z=400$. VLFW is also found in $z=600$. 

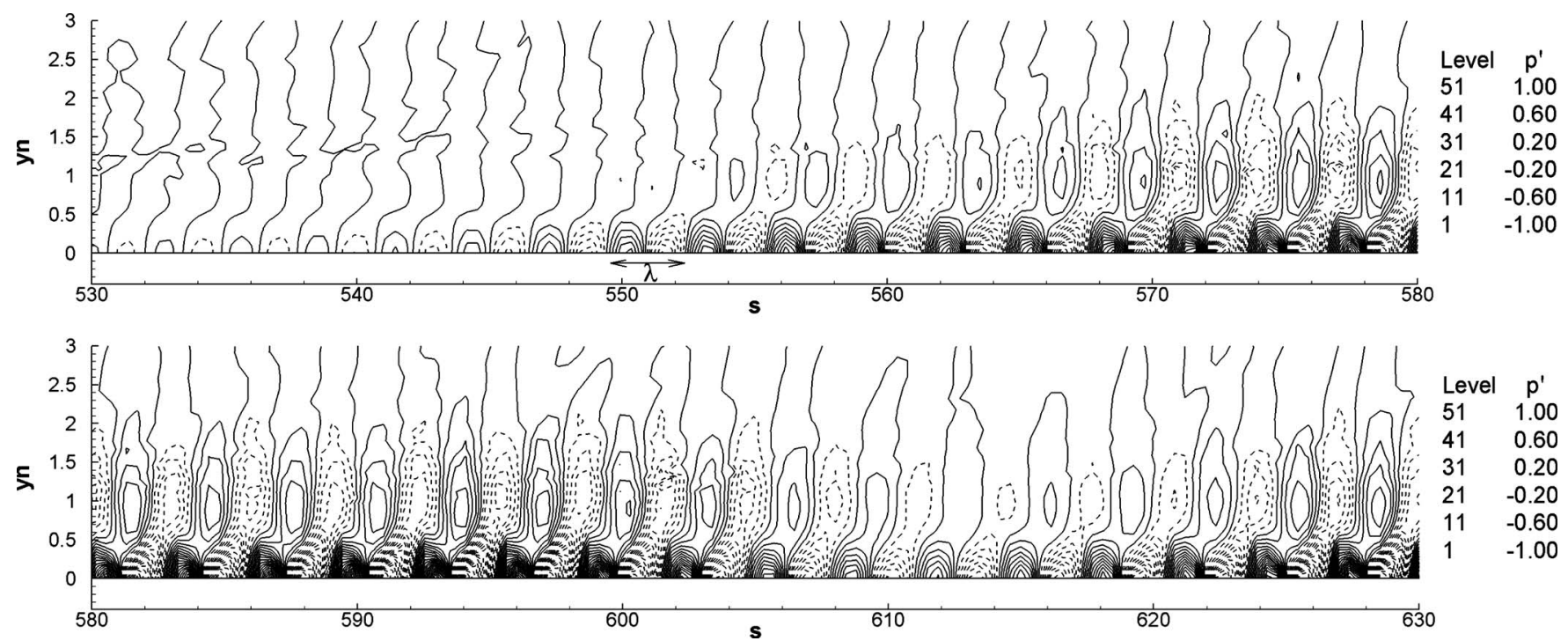

FIG. 22. Two-dimensional distribution of instantaneous disturbance pressure $p^{\prime}$ in the windward section at $t=1799.64$.

Figure 20 shows the amplitude of perturbations wave $u^{\prime}$ in the leeward section with $\omega=1.87$, which is the dominate frequency at $z=550$ [see Fig. 19(f)]. This figure also shows the receptivity stage (stage I), linear growth stage (stage II), and transition stage (stage III). This figure shows that the amplitude of wave with frequency $\omega=1.87$ keeps approximately an exponential growth in the linear growth stage. Figure 21 shows that the profile of rms pressure fluctuation $p_{\mathrm{rms}}^{\prime}$ in a wall-normal line in the leeward section with $z=550$, and the result from the LST second mode is also plotted. This figure shows that the profile of $p_{\text {rms }}^{\prime}$ is very close to the LST prediction, and indicates that the second mode perturbation is the dominate perturbation here. Figure 22 shows the twodimensional distribution of instantaneous disturbance pressure $p_{\text {DNS }}^{\prime}$ in the windward section at $t=1799.64$ of the current DNS. This figure also shows the dominating sinuouslike disturbance waves, and the wavelength of this sinuouslike wave is $\lambda_{\mathrm{DNS}} \approx 3.0$ in the region $s \approx 550$ (marked in Fig. 22 ), which is close to the local second mode's wavelength $\left(\lambda_{\mathrm{LST}} \approx 2.96\right.$ when $\left.\omega=1.87\right)$. This indicates that the second mode perturbations are dominating here.

\section{Band-pass filter analysis of the perturbation waves}

The streamwise velocity spectrum at $z=600$ [Fig. 19(g)] shows two dominate peaks: the peak for the second mode and the peak for VLFW. Characters about the second mode have been discussed above, and to further study the characters of the lower frequency component, we perform basspass filter analysis.

The filter is a hot-top filter in the frequency spectrum space as

$$
\tilde{\hat{f}}^{\prime}(\vec{r}, \omega)=G(\omega) \hat{f}^{\prime}(\vec{r}, \omega),
$$

where $\hat{f}^{\prime}$ is the frequency spectrum of disturbance $f^{\prime}$, and

$$
G(\omega)= \begin{cases}1 & \text { when } \omega_{a} \leq \omega \leq \omega_{b} \\ 0 & \text { otherwise. }\end{cases}
$$

This shows that only waves in the frequency range $\left[\omega_{a}, \omega_{b}\right]$ can pass the filter and others are cut off.

A low frequency band-pass filter $G_{L}$ with $\left[\omega_{a}, \omega_{b}\right]$ $=[0,0.4]$ and a high frequency band-pass filter $G_{H}$ with $\left[\omega_{a}, \omega_{b}\right]=[1.5,2]$ are used here. By using the filters $G_{L}$ and $G_{H}$, the low frequency component of streamwise velocity disturbance $u_{L}^{\prime}$ and high frequency one $u_{H}^{\prime}$ are computed.
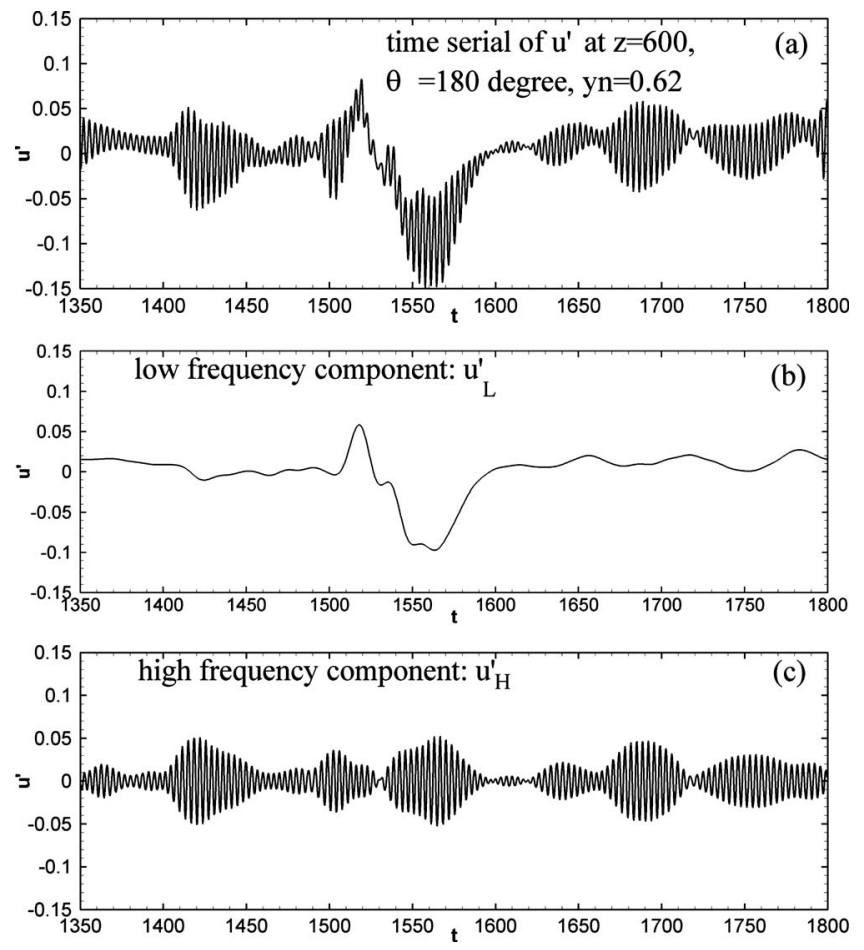

FIG. 23. Time series of streamwise velocity disturbance $u^{\prime}$ at the point $\left(z=600, \theta=180^{\circ}, y_{n}=0.62\right)$ as well as its low frequency component $u_{L}^{\prime}$ and high frequency component $u_{H}^{\prime}$. 

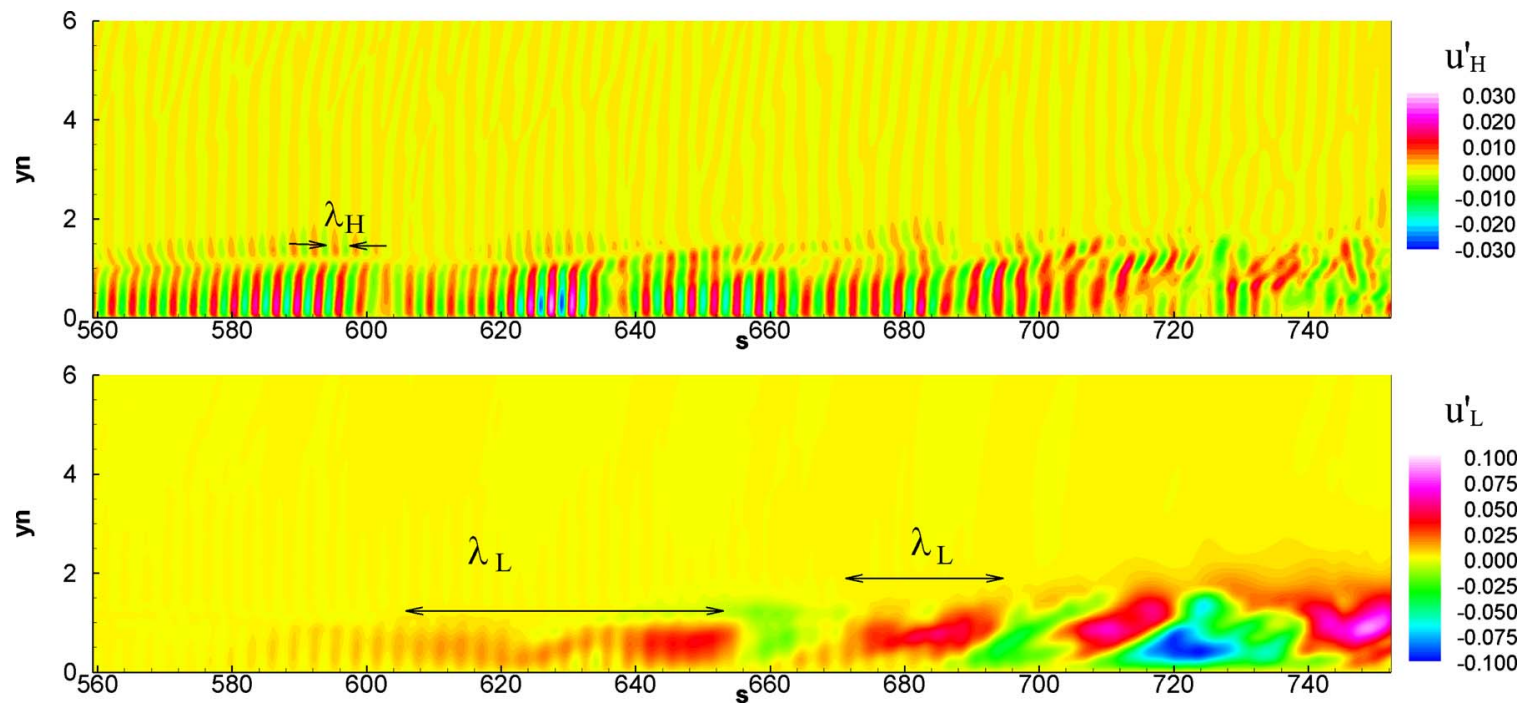

FIG. 24. (Color online) Two-dimensional distributions of high frequency component $u_{H}^{\prime}$ (top) and low frequency component $u_{L}^{\prime}$ (bottom) in the leeward section at $t=1799.64$.
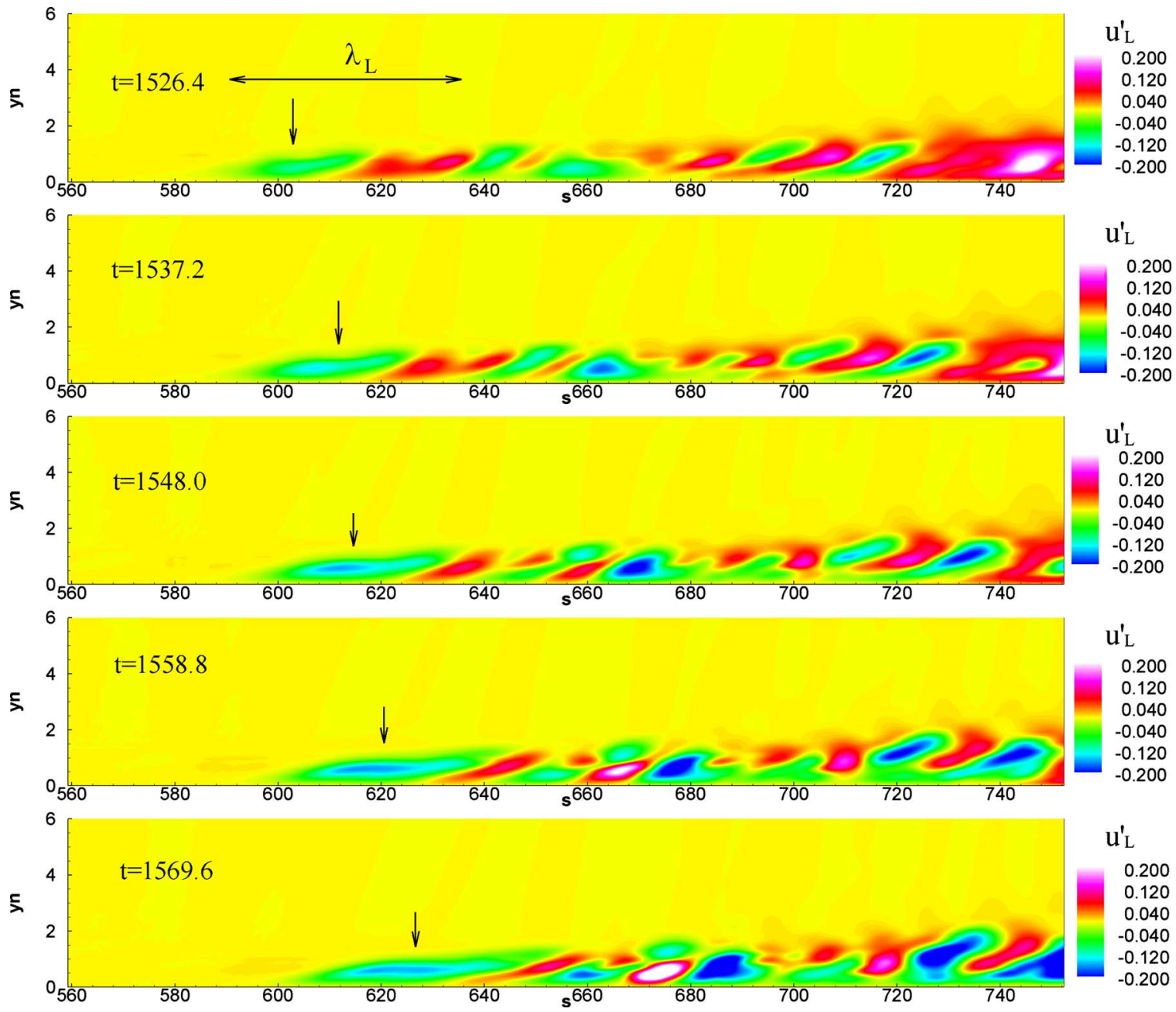

FIG. 25. (Color online) Two-dimensional distribution of low frequency component $u_{L}^{\prime}$ in the leeward section at $t=1526.4-1569.6$. 

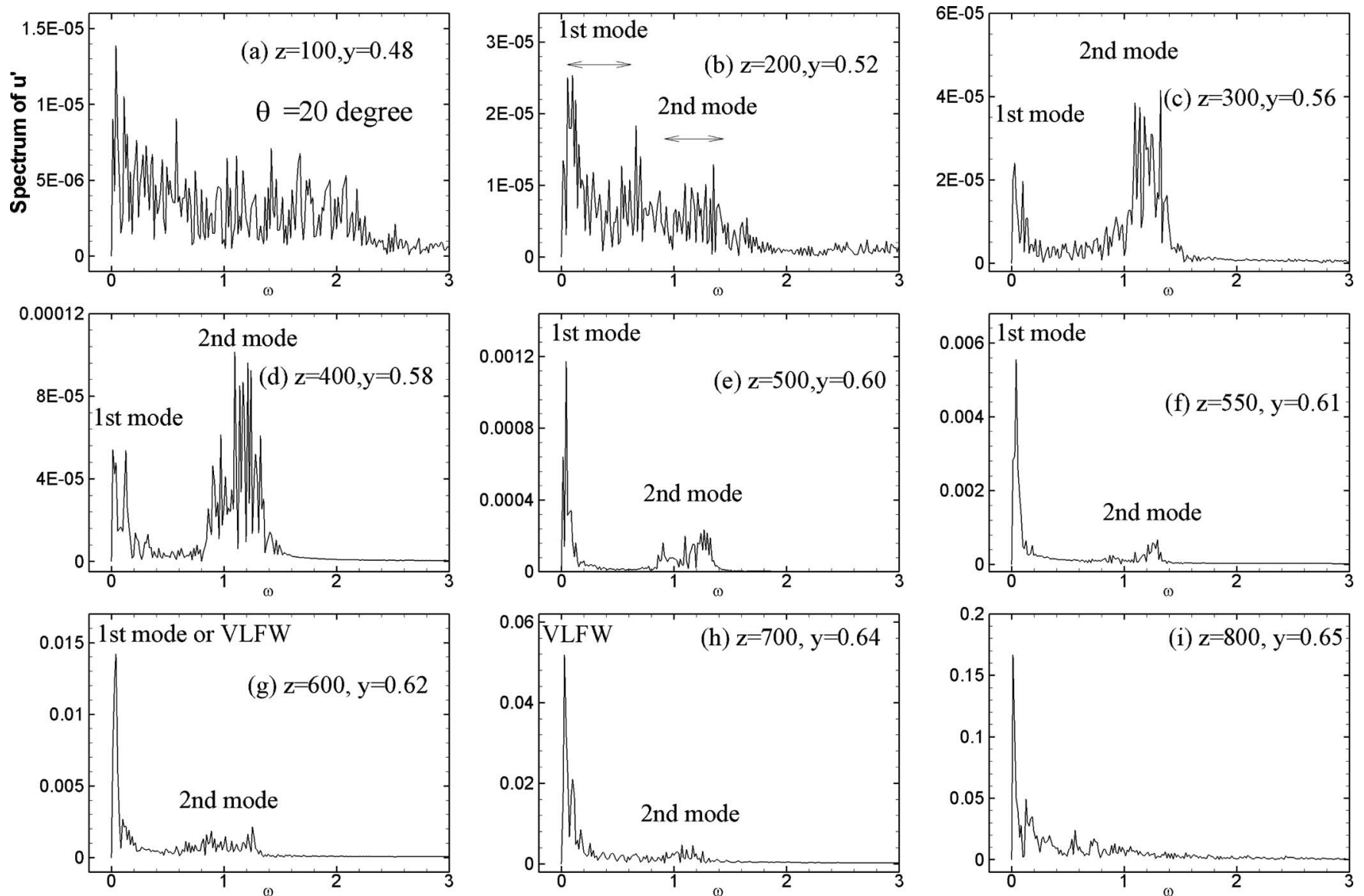

FIG. 26. Spectra of streamwise velocity fluctuations in the section $\theta=20^{\circ}$.

Figure 23 shows the time series of the streamwise velocity disturbance $u^{\prime}$ at the point $\left(z=600, \theta=180^{\circ}, y_{n}=0.62\right)$. The low frequency component $u_{L}^{\prime}$ and the high frequency component $u_{H}^{\prime}$ are also shown in this figure. This figure shows that a strong disturbance of $u_{L}^{\prime}$ occurs at $t \approx 1550$, and the spatial distribution of $u_{L}^{\prime}$ will be analyzed to study the characters of the strong disturbance.

Figure 24 shows the instantaneous two-dimensional distribution of $u_{H}^{\prime}$ and $u_{L}^{\prime}$ in the leeward section at $t=1799.64$. The high frequency component $u_{H}^{\prime}$ shows a sinuouslike structures and the wavelength is $\lambda_{H} \approx 3.0$ in the location $s \approx 600$, which is very close to the local second mode's wavelength $\left(\lambda_{\mathrm{LST}} \approx 3.0\right)$. Consider the analysis in Sec. V B 1, the high frequency components $u_{H}^{\prime}$ are mainly the second mode's disturbance. Figure 24 shows that low frequency components $u_{L}^{\prime}$ are mainly large scale structures, and the length scales are $\lambda_{L} \approx 20-50$ (approximately 1000-2500 times of the local wall unit), which is much larger than the second mode LST wave. This figure also shows that $u_{L}^{\prime}$ is stronger than $u_{H}^{\prime}$. Figure 25 shows the instantaneous two-dimensional distribution of low frequency component disturbance $u_{L}^{\prime}$ in the windward section at a time series $1526.4 \leq t \leq 1569$.6. This figure shows that a strong large scale structure (marked by arrows in this figure) passed the location $s=600$, generating the negative fluctuation peak in the time series of Fig. 23(b). The convection velocity of the low frequency large scale struc-
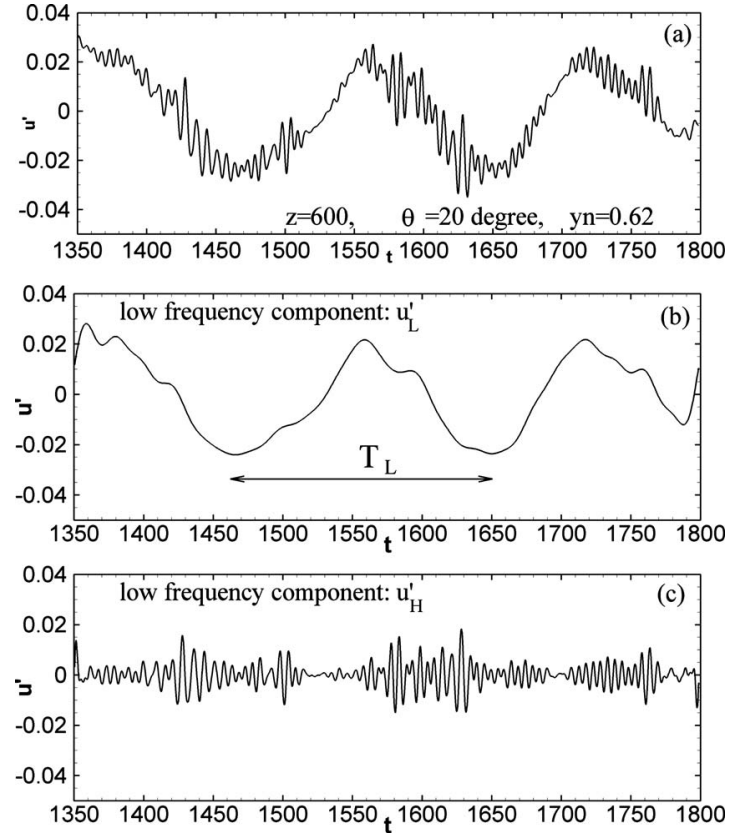

FIG. 27. Time series of streamwise velocity disturbance $u^{\prime}$ at the point $\left(z=600, \theta=20^{\circ}, y_{n}=0.62\right)$ as well as its low frequency component $u_{L}^{\prime}$ and high frequency component $u_{H}^{\prime}$. 


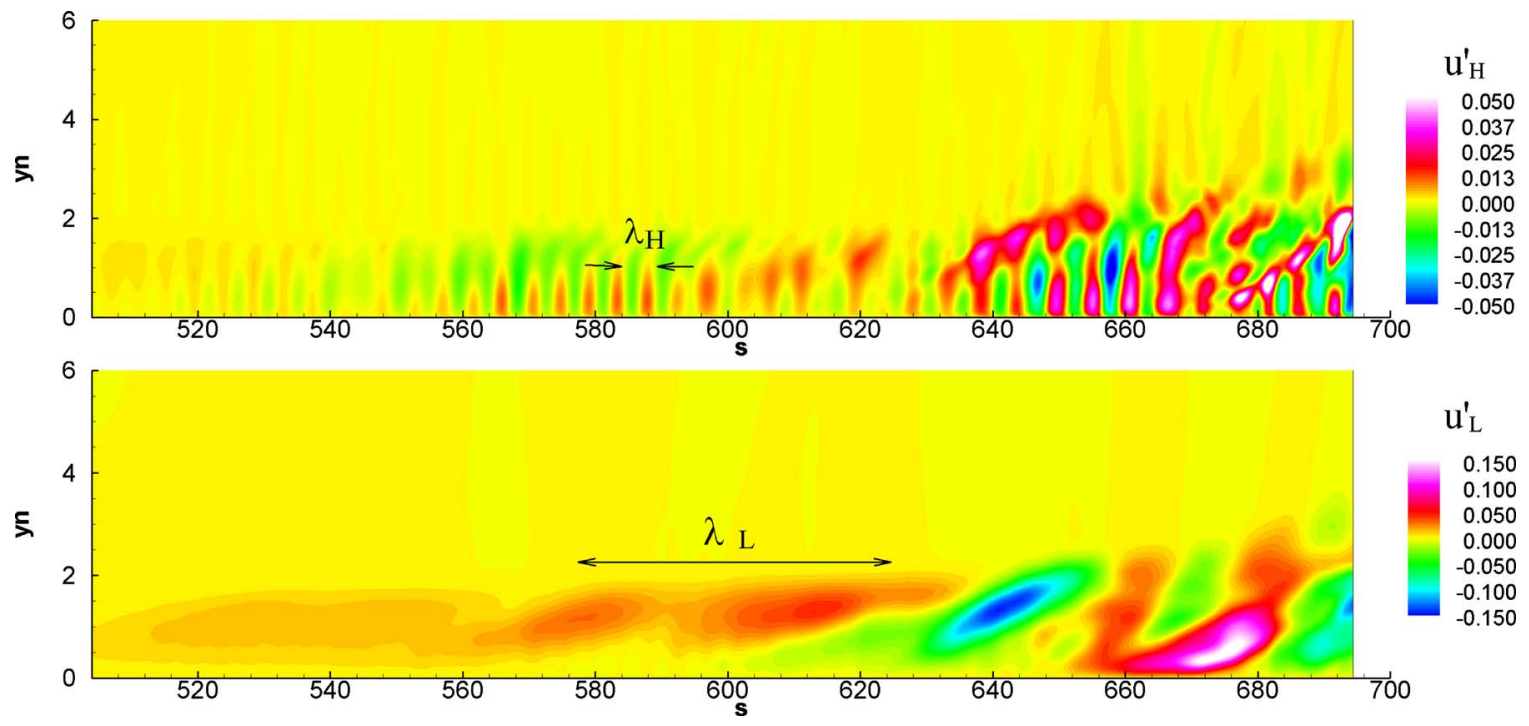

FIG. 28. (Color online) Two-dimensional distribution of high frequency component $u_{H}^{\prime}$ (top) and low frequency component $u_{L}^{\prime}$ (bottom) in the section $\theta=20^{\circ}$ at $t=1799.64$
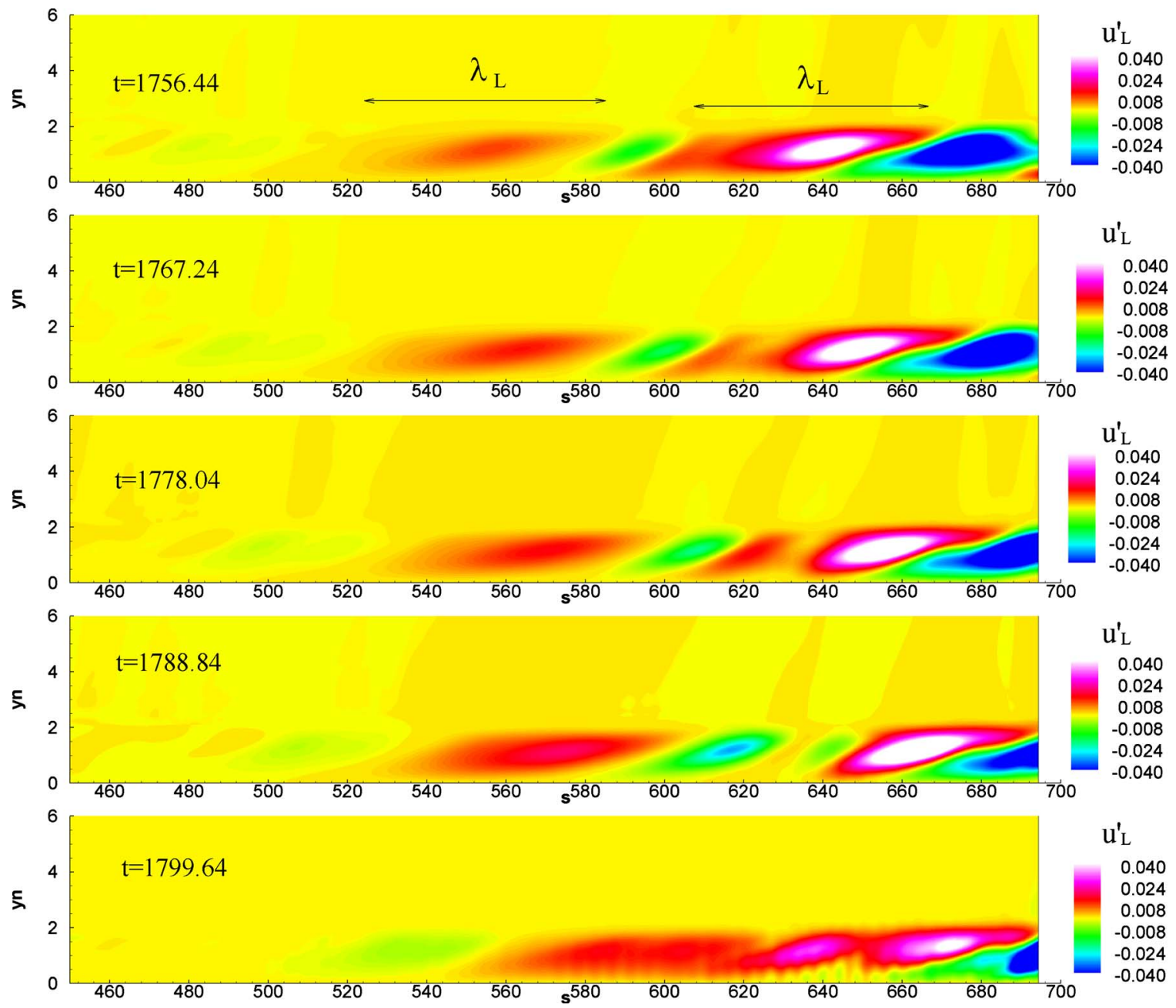

FIG. 29. (Color online) Two-dimensional distribution of low frequency component $u_{L}^{\prime}$ in the section $\theta=20^{\circ}$ at $t=1756.64-1799.64$. 
ture is approximately $0.6 U_{e}-0.7 U_{e}\left(U_{e} \approx 1\right.$ is the velocity at the boundary edge). This figure shows that the large scale structures are strongly distorted and are amplified rapidly, and indicates that they are strongly nonlinear perturbations, which are strong enough to trigger the transition.

\section{The section $\theta=20^{\circ}$}

\section{Frequency analysis}

Figure 26 shows the spectrum of streamwise velocity fluctuation in the section $\theta=20^{\circ}$. This figure shows that the high frequency components with the frequency range $\omega \in[0.5,1.4]$, which is in the unstable frequency range of the second mode, grow fast in the range $200 \leq z \leq 400$. The high frequency components are stronger than the low frequency ones $(\omega \in[0,0.3])$ at $z=300$ and $z=400$. However, as in the analysis in Sec. IV, the high frequency components' growth is suppressed in the downstream region, and the low frequency components $\omega \in[0,0.3]$ become dominate in the range $z \geq 500$. During the range $400 \leq z \leq 700$, the amplitude of low frequency streamwise velocity perturbation wave is amplified from $A \approx 5 \times 10^{-5}$ to $A \approx 5 \times 10^{-2}$, and the mean growth rate is $-\bar{\alpha}_{i}=0.023$. Figure 26(f) shows that the amplitude of the perturbation wave reaches $A \approx 0.16$ at $z=800$, where it is the start point of transition. This threshold $(A \approx 0.16)$ is higher than that in the leeward section or the windward section.

\section{Band-pass filter analysis}

To study the characters of low frequency components of perturbations in the section $\theta=20^{\circ}$, band-pass filter decomposition like that in Sec. V B 2 is performed. The low frequency filter is set as $\left[\omega_{a}, \omega_{a}\right]=[0,0.3]$ and the high frequency filter is set as $\left[\omega_{a}, \omega_{a}\right]=[0.5,1.40]$ here.

Figure 27 shows the time series of streamwise velocity disturbance $u^{\prime}$ at the point $\left(z=600, \theta=20^{\circ}, y_{n}=0.62\right)$, as well as its low frequency component $u_{L}^{\prime}$ and high frequency component $u_{H}^{\prime}$. Unlike that in the windward section [Fig. 23(b)], the low frequency component $u_{L}^{\prime}$ shows a sinuouslike wave, and indicates that it has close relationship with the linear perturbation waves. The time period of the low frequency wave is $T_{L} \approx 160$, and the frequency is $\omega_{L}=2 \pi / T_{L} \approx 0.04$. Consider that the strong first-mode LST perturbation waves are found in $z=500$ and $z=550$ [Figs. 26(e) and 26(f)], the low frequency component should be related with the firstmode LST perturbations. Figure 28 shows the instantaneous two-dimensional distribution of $u_{H}^{\prime}$ and $u_{L}^{\prime}$ in the section $\theta=20^{\circ}$ at $t=1799.64$. The high frequency component $u_{H}^{\prime}$ shows a sinuouslike structure and the wavelength is $\lambda_{H} \approx 4.0$ in the location $s \approx 600$, which is close to the local second mode's wavelength of the high frequency components. This indicates that $u_{H}^{\prime}$ are mainly the second mode's disturbance. Low frequency components $u_{L}^{\prime}$ are mainly large scale structures, and the length scales are $\lambda_{L} \approx 50-70$, which is close to the first mode's wavelength. Figure 29 shows the instantaneous two-dimensional distribution of low frequency component disturbance $u_{L}^{\prime}$ in the section $\theta=20^{\circ}$ at a time series $1756.64 \leq t \leq 1799.64$. This figure shows that the large scale structures are deformed rapidly, and they should be nonlinear perturbations, since linear wave keeps its shape during the streamwise development. Considering that the first mode wave's amplitude is much stronger than the second mode's one in the upstream [see Figs. 26(e) and 26(f)], the nonlinear perturbation must likely be triggered by the first mode's wave in this section $\theta=20^{\circ}$. So, most likely is that the VLFWs' peaks in Figs. 26(g) and 26(h) are the effects of nonlinear large scale structures triggered mainly by the upstream first mode linear waves. Both Figs. 25 and 29 show that VLFWs are very strong and are distorted rapidly downstream, and indicate that they play important roles to trigger the transition.

\section{CONCLUSION}

The DNS of boundary layer transition over a $5^{\circ}$ halfcone-angle blunt cone is performed. The free-stream Mach number is 6 and the angle of attack is $1^{\circ}$. Random wall blow and suction perturbations are used to trigger the transition. Transition locations are verified by comparing with the quiet wind tunnel experiment and the result of the $e^{N}$ theory. The following conclusions are drawn.

(1) Transition location on the cone surface shows a nonmonotonic curve, and the transition location in the range $20^{\circ} \leq \theta \leq 30^{\circ}$ is delayed significantly.

(2) The transition locations for different meridian planes are explained by using joint frequency spectrum analysis and LST. The Mack second mode instability is deemed as dominate perturbations to trigger the transition in the leeward and the windward sections. But the Mack second mode instability in the meridian planes of $20^{\circ}-30^{\circ}$ is depressed and the growth rate of the unstable waves is decreased, which leads to the delayed transition location. The frequency spectrum at $\theta=20^{\circ}$ indicates that the low frequency first mode wave is the dominate wave to trigger the transition here.

(3) VLFWs are found in the spectra just before the transition location, and the periodic time is about one order larger than the ordinary Mack second mode waves. VLFWs are large scale nonlinear perturbations, and they are triggered by the upstream first or second mode LST waves. Flow visualizations show that VLFWs are amplified and distorted rapidly during its downstream development, which indicates that VLFWs play important roles to trigger the transition.

\section{ACKNOWLEDGMENTS}

The authors thank Professor Heng Zhou, Professor Jiseng Luo, and Professor Wei Cao of Tianjin University for the LST code SAYR and their helpful comments. The authors also thank the reviewers for the helpful common suggestions, Dr. Ying Zhou of Iowa State University for polishing the English, and the Supercomputing Center of Chinese Academy of Sciences (SCCAS) and Shanghai Supercomputer Center (SSC) for providing computer time. This work was supported by the National Natural Science Foundation of China (Grant Nos. 110632050 and 10872205) and the 973 Program (Grant No. 2009CB724100). 
${ }^{1}$ L. Lees and C. C. Lin, "Investigation of the stability of the laminar boundary layer in compressible fluid," NACA Report No. TN 1115, 1946.

${ }^{2}$ L. M. Mack, "Boundary layer linear stability theory," AGARD Report No. 709, 1984.

${ }^{3}$ K. F. Stetson and R. L. Kimmel, "On hypersonic boundary layer stability," AIAA Paper No. 92-0737, 1992.

${ }^{4}$ K. F. Stetson and R. Kimmel, "On the breakdown of a hypersonic laminar boundary layer," AIAA Paper No. 93-0896, 1993.

${ }^{5}$ A. A. Maslov, S. G. Mironov, and A. A. Shiplyuk, "Hypersonic flow stability experiments," AIAA Paper No. 2002-0153, 2002.

${ }^{6}$ C. H. Su and H. Zhou, "Stability analysis and transition prediction of hypersonic boundary layer over a blunt cone with small nose bluntness at zero angle of attack," Appl. Math. Mech. 28, 563 (2007).

S. P. Schneider, "Flight data for boundary-layer transition at hypersonic and supersonic speeds," J. Spacecr. Rockets 36, 8 (1999).

${ }^{8}$ T. J. Horvath, S. A. Berry, B. R. Hollis, C. L. Chang, and B. A. Singer, "Boundary layer transition on slender cones in conventional and low disturbance Mach 6 wind tunnels," AIAA Paper No. 2002-2743, 2002.

${ }^{9}$ X. L. Zhong and Y. B. Ma, "Boundary-layer receptivity of Mach 7.99 flow over a blunt cone to free-stream acoustic waves," J. Fluid Mech. 556, 55 (2006).

${ }^{10} \mathrm{C}$. H. Su and H. Zhou, "Transition prediction of a hypersonic boundary layer over a cone at small angle of attack-with the improvement of $e^{N}$ method," Sci. China, Ser. G 52, 115 (2009).

${ }^{11}$ P. Moin and K. Mahes, "Direct numerical simulation: A tool in turbulence research," Annu. Rev. Fluid Mech. 30, 539 (1998).

${ }^{12}$ K. F. Stetson, "Hypersonic boundary layer transition experiments," Report No. AFWAL-TR-80-3062, 1980

${ }^{13}$ X. L. Li, D. X. Fu, and Y. W. Ma, "Direct numerical simulation of boundary layer transition over a blunt cone," AIAA J. 46, 2899 (2008).

${ }^{14}$ G. S. Jiang and C. W. Shu, "Efficient implementation of weighted ENO schemes," J. Comput. Phys. 126, 202 (1996).

${ }^{15} \mathrm{~N}$. A. Adams, "Direct simulation of the turbulent boundary layer along a compression ramp at $M=3$ and $R e=1685$," J. Fluid Mech. 420, 47 (2000).

${ }^{16}$ X. L. Li, D. X. Fu, and Y. W. Ma, "DNS of compressible turbulent boundary layer over a blunt wedge," Sci. China, Ser. G 48, 129 (2005).

${ }^{17}$ X. L. Li, D. X. Fu, and Y. W. Ma, "DNS of compressible turbulent boundary layer around a sharp cone," Sci. China, Ser. G 51, 699 (2008).

${ }^{18}$ X. L. Li, D. X. Fu, Y. W. Ma, and H. Gao, "Acoustic calculation for supersonic turbulent boundary layer flow," Chin. Phys. Lett. 26, 094701 (2009).

${ }^{19}$ S. Pirozzoli and F. Grasso, "Direct numerical simulation and analysis of a spatially evolving supersonic turbulent boundary layer at $M=225$," Phys. Fluids 16, 530 (2004) 